\title{
Brunnian braids on surfaces
}

\author{
VALERY G BARDAKOV \\ ROMAN MIKHAILOV \\ VLADIMIR V VERSHININ \\ JIE WU
}

\begin{abstract}
We determine a set of generators for the Brunnian braids on a general surface $M$ for $M \neq S^{2}$ or $\mathbb{R} \mathrm{P}^{2}$. For the case $M=S^{2}$ or $\mathbb{R} \mathrm{P}^{2}$, a set of generators for the Brunnian braids on $M$ is given by our generating set together with the homotopy groups of a 2-sphere.
\end{abstract}

57M07, 57M99; 20F36, 55Q40

\section{Introduction}

Let $M$ be a compact connected surface, possibly with boundary, and let $B_{n}(M)$ denote the $n$-strand braid group on a surface $M$. From the point of view of braids, compactness of a surface is not essential: braids stay the same if you replace a boundary component by a puncture. However the number of punctures must be finite, so that the fundamental group and the braid groups will be finitely generated.

A Brunnian braid means a braid that becomes trivial after removing any one of its strands. The formal definition of Brunnian braids is given in Section 2. A typical example of a 3-strand Brunnian braid on a disk is the braid given by the expression $\left(\sigma_{1}^{-1} \sigma_{2}\right)^{3}$, where $\sigma_{1}$ and $\sigma_{2}$ are the standard generators of the 3-strand braid group $\left\langle\sigma_{1}, \sigma_{2} \mid \sigma_{1} \sigma_{2} \sigma_{1}=\sigma_{2} \sigma_{1} \sigma_{2}\right\rangle$.

Let $\operatorname{Brun}_{n}(M)$ denote the set of the $n$-strand Brunnian braids. Then $\operatorname{Brun}_{n}(M)$ forms a subgroup of $B_{n}(M)$. A classical question proposed by GS Makanin [19] in 1980 is to determine a set of generators for Brunnian braids over the disk. Brunnian braids were called smooth braids by Makanin. This question was answered by D L Johnson [12] and G G Gurzo [11]. J Y Li and J Wu [16; 23] gave different approach to this question. In the 1970s, H Levinson [14; 15] defined a notion of $k$-decomposable braid, which becomes trivial after removal of any arbitrary $k$ strings. In his terminology a decomposable braid means 1-decomposable and therefore, Brunnian. 
A J Berrick, F R Cohen, Y L Wong and J Wu [2] gave a connection between Brunnian braids and the homotopy groups of spheres. In particular, the exact sequence

$$
1 \rightarrow \operatorname{Brun}_{n+1}\left(S^{2}\right) \rightarrow \operatorname{Brun}_{n}\left(D^{2}\right) \rightarrow \operatorname{Brun}_{n}\left(S^{2}\right) \rightarrow \pi_{n-1}\left(S^{2}\right) \rightarrow 1
$$

was proved for $n>4$.

J Birman [3, Question 23, page 219] asked how to determine a free basis for the intersection $\operatorname{Brun}_{n}\left(D^{2}\right) \cap R_{n-1}$ where

$$
R_{n-1}=\operatorname{Ker}\left(B_{n}\left(D^{2}\right) \rightarrow B_{n}\left(S^{2}\right)\right) .
$$

Her motivation was that the kernel of the Gassner representation is a subgroup of $\operatorname{Brun}_{n}\left(D^{2}\right) \cap R_{n-1}$. From the exact sequence (1-1) it follows that Birman's question, for $n>5$, is about a free basis of Brunnian braids over the sphere $S^{2}$. As far as we know this question remains open.

The purpose of this article is to determine a set of generators for $\operatorname{Brun}_{n}(M)$ for a general surface $M$. We are able to determine a generating set for $\operatorname{Brun}_{n}(M)$ except in two special cases, where $M=S^{2}$ or $\mathbb{R P}^{2}$. For the case $M=S^{2}$ or $\mathbb{R P}^{2}$, we are able to determine a generating set for a (normal) subgroup of $\operatorname{Brun}_{n}(M)$, with the factor group given by $\pi_{n-1}\left(S^{2}\right)$.

Recall the notion of the symmetric commutator product (see $\mathrm{Li}$ and $\mathrm{Wu}$ [17] and Mikhailov, Passi and $\mathrm{Wu}$ [20]). Given a group $G$, and a set of normal subgroups $R_{1}, \ldots, R_{n}(n \geq 2)$, the symmetric commutator product of these subgroups is defined as

$$
\left[R_{1}, \ldots, R_{n}\right]_{S}:=\prod_{\sigma \in \Sigma_{n}}\left[\left[R_{\sigma(1)}, R_{\sigma(2)}\right], \ldots, R_{\sigma(n)}\right]
$$

where $\Sigma_{n}$ is the symmetric group of degree $n$.

Let $P_{n}(M)$ be the $n$-strand pure braid group on $M$. Let $D^{2}$ be a small disk in $M$. Then the inclusion $f: D^{2} \hookrightarrow M$ induces a group homomorphism

$$
f_{*}: P_{n}\left(D^{2}\right) \longrightarrow P_{n}(M) \text {. }
$$

Recall that the pure Artin braid group $P_{n}\left(D^{2}\right)$ is a subgroup of the braid group

$$
\begin{array}{r}
B_{n}=\left\langle\sigma_{1}, \ldots, \sigma_{n-1}\right| \sigma_{i} \sigma_{i+1} \sigma_{i}=\sigma_{i+1} \sigma_{i} \sigma_{i+1}, i=1, \ldots, n-2, \\
\left.\sigma_{i} \sigma_{j}=\sigma_{j} \sigma_{i},|i-j| \geq 2\right\rangle .
\end{array}
$$

generated by the elements

$$
A_{i, j}=\sigma_{j-1} \sigma_{j-2} \cdots \sigma_{i+1} \sigma_{i}^{2} \sigma_{i+1}^{-1} \cdots \sigma_{j-2}^{-1} \sigma_{j-1}^{-1},
$$


for $1 \leq i<j \leq n$. Let $A_{i, j}[M]=f_{*}\left(A_{i, j}\right)$ and let $\left\langle\left\langle A_{i, j}[M]\right\rangle\right\rangle^{P}$ be the normal closure of $A_{i, j}[M]$ in $P_{n}(M)$. Note that a set of generators for $\left\langle\left\langle A_{i, j}[M]\right\rangle\right\rangle^{P}$ is given by $\beta A_{i, j}[M] \beta^{-1}$ for $\beta \in P_{n}(M)$. Thus a set of generators for the iterated subgroup

$$
\left.\left[\left\langle A_{1, n}[M]\right\rangle\right\rangle^{P},\left\langle\left\langle A_{2, n}[M]\right\rangle\right\rangle^{P}, \ldots,\left\langle\left\langle A_{n-1, n}[M]\right\rangle\right\rangle^{P}\right]_{S}
$$

can be given.

Now we compute $\operatorname{Brun}_{n}(M)$ as follows.

Theorem 1.1 Let $M$ be a connected 2-manifold and let $n \geq 2$. Let

$$
R_{n}(M)=\left[\left\langle\left\langle A_{1, n}[M]\right\rangle\right\rangle^{P},\left\langle\left\langle A_{2, n}[M]\right\rangle\right\rangle^{P}, \ldots,\left\langle\left\langle A_{n-1, n}[M]\right\rangle\right\rangle^{P}\right]_{S} .
$$

(1) If $M \neq S^{2}$ or $\mathbb{R P}^{2}$, then

$$
\operatorname{Brun}_{n}(M)=R_{n}(M) .
$$

(2) If $M=S^{2}$ and $n \geq 5$, then there is a short exact sequence

$$
R_{n}\left(S^{2}\right) \hookrightarrow \operatorname{Brun}_{n}\left(S^{2}\right) \rightarrow \pi_{n-1}\left(S^{2}\right) .
$$

(3) If $M=\mathbb{R} \mathrm{P}^{2}$ and $n \geq 4$ then there is a short exact sequence

$$
R_{n}\left(\mathbb{R} \mathrm{P}^{2}\right) \hookrightarrow \operatorname{Brun}_{n}\left(\mathbb{R} \mathrm{P}^{2}\right) \rightarrow \pi_{n-1}\left(S^{2}\right) .
$$

Remark (1) Assertion (2) fails for $n=3$, 4. A free basis for $\operatorname{Brun}_{4}\left(S^{2}\right)$ was given in [2]. Assertion (3) fails for $n=2,3$. For the cases $n \leq 3$, our result is given in Propositions 3.3, 3.6 and 4.9 by explicit computations.

(2) In the classical case where $M=D^{2}$, assertion (1) gives a better format for answering Makanin's question as we describe Brunnian braids as an explicit iterated commutator subgroup. In this case the assertion was proved in [17]. Assertion (2) was essentially given in [2, Theorem 1.2]. Here we give an explicit determination for the kernel of $\operatorname{Brun}_{n}\left(S^{2}\right) \rightarrow \pi_{n-1}\left(S^{2}\right)$ for $n \geq 5$. Assertion (3) gives a new connection between Brunnian braids and homotopy groups. The first case in assertion (3) $(n=4)$ is that the Hopf map $S^{3} \rightarrow S^{2}$ lifts to a 4 -strand Brunnian braid on $\mathbb{R} \mathrm{P}^{2}$.

(3) For the classical case, the inclusion

$$
R_{n}\left(D^{2}\right) \hookrightarrow \operatorname{Brun}_{n}\left(D^{2}\right)
$$

was observed by Levinson [15, page 53]. 
By Corollary 2.5, $\operatorname{Brun}_{n}(M)$ is a normal subgroup of $B_{n}(M)$ for $n \geq 3$. As an abstract group, $\operatorname{Brun}_{n}(M)$ is a free group of infinite rank for $n \geq 3$ with $M \neq S^{2}$ or $\mathbb{R P}{ }^{2}$, for $n \geq 5$ with $M=S^{2}$ and for $n \geq 4$ with $M=\mathbb{R P}^{2}$. A natural question is whether the factor group $B_{n}(M) / \operatorname{Brun}_{n}(M)$ is finitely presented. Our answer to this question is positive.

Theorem 1.2 Let $M$ be a connected compact 2-manifold. Then the factor groups $P_{n}(M) / \operatorname{Brun}_{n}(M)$ and $B_{n}(M) / \operatorname{Brun}_{n}(M)$ are finitely presented for each $n \geq 3$.

The article is organized as follows. In Section 2, we give a review on Brunnian braids. The determination of a generating set for Brunnian braids is given in Section 3. In Section 4, we compute the 3-strand Brunnian braids on the projective plane. The proof of Theorem 1.2 is given in Section 5. In Section 6, we give an algorithm for determining a free basis for Brunnian Braids. In the Appendix we prove the technical results stated in Section 4.

\section{Brunnian braids}

\subsection{Configuration spaces and the braid groups}

Let $M$ be a topological space and let $M^{n}$ be the $n$-fold Cartesian product of $M$. The $n$-th ordered configuration space, $F(M, n)$ is defined by

$$
F(M, n)=\left\{\left(x_{1}, \ldots, x_{n}\right) \in M^{n} \mid x_{i} \neq x_{j} \text { for } i \neq j\right\}
$$

with the subspace topology on $M^{n}$. The symmetric group $\Sigma_{n}$ acts on $F(M, n)$ by permuting coordinates. The orbit space

$$
B(M, n)=F(M, n) / \Sigma_{n}
$$

is called the $n$-th unordered configuration space. The braid group $B_{n}(M)$ is defined to be the fundamental group $\pi_{1}(B(M, n))$. The pure braid group $P_{n}(M)$ is defined to be the fundamental group $\pi_{1}(F(M, n))$. From the covering $F(M, n) \rightarrow F(M, n) / \Sigma_{n}$, there is a short exact sequence of groups

$$
1 \rightarrow P_{n}(M) \rightarrow B_{n}(M) \rightarrow \Sigma_{n} \rightarrow 1 .
$$

A geometric description of the elements in $B_{n}(M)$ can be given as follows. Let $\left(q_{1}, \ldots, q_{n}\right)$ be the basepoint of $F(M, n)$ and let

$$
p: F(M, n) \rightarrow F(M, n) / \Sigma_{n}
$$


be the quotient map. The basepoint of $F(M, n) / \Sigma_{n}$ is chosen to be $p\left(q_{1}, \ldots, q_{n}\right)$. Let $[\lambda]$ be an element in $\pi_{1}\left(F(M, n) / \Sigma_{n}\right)$ represented by a loop $\lambda: S^{1} \rightarrow F(M, n) / \Sigma_{n}$. Since

$$
p: F(M, n) \rightarrow F(M, n) / \Sigma_{n}
$$

is a covering, the loop $\lambda$ lifts to a unique path $\tilde{\lambda}$ : $[0,1] \rightarrow F(M, n)$ starting from $\tilde{\lambda}(0)=\left(q_{1}, \ldots, q_{n}\right)$ and ending with $\tilde{\lambda}(1)=\left(q_{\sigma(1)}, \ldots, q_{\sigma(n)}\right)$ for some $\sigma \in \Sigma_{n}$. Let

$$
\tilde{\lambda}(t)=\left(\tilde{\lambda}_{1}(t), \ldots, \tilde{\lambda}_{n}(t)\right) \in F(M, n) \subseteq M^{n} .
$$

Then $\tilde{\lambda}_{i}(t) \neq \tilde{\lambda}_{j}(t)$ for $i \neq j$ and any $0 \leq t \leq 1$. The strands

$$
\left\{\left(\tilde{\lambda}_{i}(t), t\right) \mid 1 \leq i \leq n\right\}
$$

in the cylinder $M \times[0,1]$ give the intuitive braided description of $\lambda$. The precise definition of geometric braids is as follows.

Let $\left\{p_{1}, p_{2}, \ldots, p_{n}\right\}$ be $n$ distinct points in $M$. Consider the cylinder $M \times I$. A geometric braid

$$
\rho=\left\{\rho_{1}, \ldots, \rho_{n}\right\}
$$

at the basepoints $\left\{p_{1}, \ldots, p_{n}\right\}$ is a collection of $n$ paths in the cylinder $M \times I$ such that $\rho_{i}(t)=\left(\lambda_{i}(t), t\right)$ and

(1) $\lambda_{1}(0)=p_{1}, \ldots, \lambda_{n}(0)=p_{n}$;

(2) $\lambda_{1}(1)=p_{\sigma(1)}, \ldots, \lambda_{n}(1)=p_{\sigma(n)}$ for some $\sigma \in \Sigma_{n}$;

(3) $\lambda_{i}(t) \neq \lambda_{j}(t)$ for $0 \leq t \leq 1$ and $i \neq j$.

Let $\rho=\left\{\rho_{1}, \ldots, \rho_{n}\right\}$ and $\rho^{\prime}=\left\{\rho_{1}^{\prime}, \ldots, \rho_{n}^{\prime}\right\}$ be two geometric braids. We say that $\rho$ is equivalent to $\rho^{\prime}$, denoted by $\rho \sim \rho^{\prime}$, if there exists a continuous sequence of geometric braids

$$
\rho^{s}=\left(\lambda^{s}, t\right)=\left\{\left(\lambda_{1}^{s}(t), t\right), \ldots,\left(\lambda_{n}^{s}(t), t\right)\right\}, \quad 0 \leq s \leq 1
$$

such that

(1) $\lambda_{1}^{s}(0)=p_{1}, \ldots, \lambda_{n}^{s}(0)=p_{n}$ for each $0 \leq s \leq 1$;

(2) $\lambda_{1}^{s}(1)=\lambda_{1}^{0}(1), \ldots, \lambda_{n}^{s}(1)=\lambda_{n}^{0}(1)$ for each $0 \leq s \leq 1$;

(3) $\lambda^{0}=\lambda$ and $\lambda^{1}=\lambda^{\prime}$.

In other words $\rho \sim \rho^{\prime}$ if and only if they represent the same path homotopy class in the configuration space $F(M, n)$. We also use the term geometric braid to mean an equivalence class of geometric braids. 
The product of two geometric braids $\beta$ and $\beta^{\prime}$ is defined to be the composition of the strands. More precisely, let $\beta$ be represented by $\rho=\left\{\rho_{1}, \ldots, \rho_{n}\right\}$ with $\rho_{1}(1)=$ $p_{\sigma(1)}, \ldots, \rho_{n}(1)=p_{\sigma(n)}$ and let $\beta^{\prime}$ be represented by $\rho^{\prime}=\left\{\rho_{1}^{\prime}, \ldots, \rho_{n}^{\prime}\right)$. Then the product $\beta \beta^{\prime}$ is represented by

$$
\rho * \rho^{\prime}=\left\{\rho_{1} * \rho_{\sigma(1)}^{\prime}, \ldots, \rho_{n} * \rho_{\sigma(n)}^{\prime}\right\},
$$

where $\rho_{i} * \rho_{\sigma(i)}^{\prime}$ is the path product.

\subsection{Removing strands}

A simple (half-open) curve in a space $M$ is a continuous injection $\theta: \mathbb{R}^{+}=[0, \infty) \rightarrow M$. The distinct points $\left\{p_{1}, \ldots, p_{n}\right\}$ in $M$ are said to be well-ordered with respect to a simple curve $\theta$ if there exist points $t_{i} \in[0,1]$ with $0 \leq t_{1}<t_{2}<\cdots<t_{n}$ such that $p_{i}=\theta\left(t_{i}\right)$ for $1 \leq i \leq n$.

Let $\mathbf{p}=\left(p_{1}, \ldots, p_{n}\right)$ and $\mathbf{p}^{\prime}=\left(p_{1}^{\prime}, \ldots, p_{n}^{\prime}\right)$ be two sets of $n$ distinct well-ordered points with respect to $\theta$ with $p_{i}=\theta\left(t_{i}\right)$ and $p_{i}^{\prime}=\theta\left(t_{i}^{\prime}\right)$. Define

$$
L\left(\mathbf{p}, \mathbf{p}^{\prime}\right)(s)=\left\{L\left(\mathbf{p}, \mathbf{p}^{\prime}\right)_{i}(s)=\theta\left((1-s) t_{i}+s t_{i}^{\prime}\right) \mid 1 \leq i \leq n\right\}
$$

for $0 \leq s \leq 1 ; L\left(\mathbf{p}, \mathbf{p}^{\prime}\right)(s) \in M^{n}$. Observe that, for each $1 \leq i<j \leq n$ and $0 \leq s \leq 1$,

$$
(1-s) t_{i}+s t_{i}^{\prime}<(1-s) t_{j}+s t_{j}^{\prime}
$$

as $t_{i}<t_{j}$ and $t_{i}^{\prime}<t_{j}^{\prime}$. So $L\left(\mathbf{p}, \mathbf{p}^{\prime}\right)(s)$ is a set of $n$ distinct well-ordered points with respect to $\theta$ for $0 \leq s \leq 1$.

Now let $\mathbf{p}=\left(p_{1}, \ldots, p_{n}\right)$ and $\mathbf{p}^{\prime}=\left(p_{1}^{\prime}, \ldots, p_{n}^{\prime}\right)$ be two sets of $n$ distinct points on the curve $\theta$. There exist unique permutations $\sigma, \tau \in \Sigma_{n}$ such that

$$
\mathbf{p}_{\sigma}=\left(p_{\sigma(1)}, \ldots, p_{\sigma(n)}\right) \text { and } \mathbf{p}_{\tau}^{\prime}=\left(p_{\tau(1)}^{\prime}, \ldots, p_{\tau(n)}^{\prime}\right)
$$

are well-ordered with respect to $\theta$. We call

$$
L\left(\mathbf{p}_{\sigma}, \mathbf{p}_{\tau}^{\prime}\right)^{\sigma^{-1}}\left\{L\left(\mathbf{p}_{\sigma}, \mathbf{p}_{\tau}^{\prime}\right)_{\sigma^{-1}(i)} \mid 1 \leq i \leq n\right\}
$$

an $n$-strand $\theta$-linear braid from $\mathbf{p}$ to a permutation of $\mathbf{p}^{\prime}$.

Let $M$ be a space with a simple curve $\theta$ and let the basepoints $\left(p_{1}, p_{2}, \ldots, p_{n}\right)$ of the braids on $M$ be well-ordered with respect to $\theta$. The system of removing strands $d_{i}: B_{n}(M) \rightarrow B_{n-1}(M)$ is defined as follows:

Definition Let $\beta \in B_{n}(M)$ be a braid represented by $\lambda=\left\{\lambda_{1}, \ldots, \lambda_{n}\right\}$ with

$$
\lambda_{1}(1)=p_{\sigma(1)}, \ldots, \lambda_{n}(1)=p_{\sigma(n)} .
$$


Then the braid $d_{i}(\beta)$ is defined to be the equivalence class represented by the path product of the strands given by

$$
L *\left\{\lambda_{1}, \ldots, \lambda_{i-1}, \lambda_{i+1}, \ldots, \lambda_{n}\right\} * L^{\prime},
$$

where $L$ is the $\theta$-linear braid from $\left(p_{1}, \ldots, p_{n-1}\right)$ to $\left(p_{1}, \ldots, p_{i-1}, p_{i+1}, \ldots, p_{n}\right)$, and $L^{\prime}$ is the $\theta$-linear braid from $\left(p_{\sigma(1)}, \ldots, p_{\sigma(i-1)}, p_{\sigma(i+1)}, \ldots, p_{\sigma(n)}\right)$ to a permutation of $\left(p_{1}, \ldots, p_{n-1}\right)$.

It follows from this definition that the operation $d_{i}$ does not depend on the choice of $\lambda$ in the class $\beta$. Intuitively, the operation $d_{i}: B_{n}(M) \rightarrow B_{n-1}(M)$ is obtained by forgetting the $i$-th strand and gluing back to the fixed choice of the basepoints using $\theta$-linear braids.

From now on we always assume that the space $M$ has a simple curve $\theta$ and that the basepoints of the braids on $M$ are located on the curve $\theta$ starting with a set $\mathbf{p}$ of well-ordered points with respect to $\theta$ and ending with a permutation on $\mathbf{p}$. Recall that there is a short exact sequence

$$
1 \rightarrow P_{n}(M) \rightarrow B_{n}(M) \rightarrow \Sigma_{n} \rightarrow 1 .
$$

The braid group $B_{n}(M)$ acts on the right on the letters $\{1,2, \ldots, n\}$ through the epimorphism $B_{n}(M) \rightarrow \Sigma_{n}$, which can be described as follows. Let $\beta$ be represented by an $n$-strand geometric braid

$$
\lambda=\left\{\lambda_{i}(t) \mid 1 \leq i \leq n\right\}
$$

with $\lambda_{i}(0)=p_{i}$. Then $i \cdot \beta$ is given by the formula

$$
\lambda_{i}(1)=p_{i \cdot \beta}
$$

for $1 \leq i \leq n$.

Proposition 2.1 [2, Proposition 4.2.1(1)] Let $M$ be a space with a simple curve. Then the operations

$$
d_{i}: B_{n}(M) \rightarrow B_{n-1}(M), \quad 1 \leq i \leq n,
$$

satisfy the following identities:

(1) $d_{i} d_{j}=d_{j} d_{i+1}$ for $i \geq j$.

(2) $d_{i}\left(\beta \beta^{\prime}\right)=d_{i}(\beta) d_{i \cdot \beta}\left(\beta^{\prime}\right)$.

Corollary 2.2 The map $d_{i}$ is homomorphism when restricted to the pure braid group $P_{n}(M)$. 
Note In [2], the removing-strand operations are labeled by $d_{0}, \ldots, d_{n-1}$ to coincide with simplicial terminology. The above identities are directly translated from [2, Proposition 4.2.1(1)].

\subsection{Brunnian braids}

Definition 2.3 Let $M$ be a space with a simple curve. A braid $\beta \in B_{n}(M)$ is called Brunnian if $d_{i}(\beta)=1$ for each $1 \leq i \leq n$. The set of $n$-strand Brunnian braids is denoted by $\operatorname{Brun}_{n}(M)$. For convention, any $1-$ strand braid is regarded as a Brunnian braid.

Intuitively a Brunnian braid means a braid that becomes trivial after removing any one of its strands. If $\beta, \beta^{\prime} \in \operatorname{Brun}_{n}(M)$, then

$$
d_{i}\left(\beta \beta^{\prime}\right)=d_{i}(\beta) d_{i \cdot \beta}\left(\beta^{\prime}\right)=1
$$

for $1 \leq i \leq n$ and so the product $\beta \beta^{\prime} \in \operatorname{Brun}_{n}(M)$. Similar $\beta^{-1}$ is Brunnian provided $\beta$ is. Thus $\operatorname{Brun}_{n}(M)$ is a subgroup of $B_{n}(M)$.

Proposition 2.4 Suppose $M$ is a space with a simple curve. Then the subgroup $\operatorname{Brun}_{n}(M) \cap P_{n}(M)$ is normal in $B_{n}(M)$ for each $n \geq 1$.

Proof Let $\beta \in \operatorname{Brun}_{n}(M) \cap P_{n}(M)$ and let $\gamma \in B_{n}(M)$. Then

$$
\begin{aligned}
d_{i}\left(\gamma \beta \gamma^{-1}\right) & =d_{i}(\gamma \beta) d_{i \cdot(\gamma \beta)}\left(\gamma^{-1}\right) \\
& =d_{i}(\gamma) d_{i \cdot \gamma}(\beta) d_{i \cdot(\gamma \beta)}\left(\gamma^{-1}\right) \\
& =d_{i}(\gamma) d_{i \cdot(\gamma \beta)}\left(\gamma^{-1}\right)
\end{aligned}
$$

for $1 \leq i \leq n$. Since $\beta \in P_{n}(M)$, the elements $\gamma$ and $\gamma \beta$ have the same image in $\Sigma_{n}=B_{n}(M) / P_{n}(M)$ and so $i \cdot(\gamma \beta)=i \cdot \gamma$. The assertion follows from the equation

$$
1=d_{i}(1)=d_{i}\left(\gamma \gamma^{-1}\right)=d_{i}(\gamma) d_{i \cdot \gamma}\left(\gamma^{-1}\right)=d_{i}(\gamma) d_{i \cdot(\gamma \beta)}\left(\gamma^{-1}\right) .
$$

Corollary 2.5 Let $M$ be a space with a simple curve. Then $\operatorname{Brun}_{n}(M)$ is a normal subgroup of $B_{n}(M)$ for $n \geq 3$.

Proof According to [2, Proposition 4.2.2], $\operatorname{Brun}_{n}(M) \leq P_{n}(M)$ for $n \geq 3$ and hence the result.

The case $n=2$ is exceptional, since Corollary 2.5 does not hold in this case. 
Proposition 2.6 Let $M$ be a connected 2-manifold. Then $\operatorname{Brun}_{2}(M)$ is a normal subgroup of $B_{2}(M)$ if and only if $\pi_{1}(M)=\{1\}$.

Proof If $\pi_{1}(M)=\{1\}$, then $B_{2}(M)=\operatorname{Brun}_{2}(M)$ as $B_{1}(M)=\pi_{1}(M)$.

Suppose that $\pi_{1}(M) \neq\{1\}$. Let $D^{2}$ be a small disk in $M \backslash \partial M$. The inclusion $f: D^{2} \rightarrow M$ induces canonical maps

$$
(f, f): F\left(D^{2}, 2\right) \succ F(M, 2) \quad \text { and } \quad(f, f): F\left(D^{2}, 2\right) / \Sigma_{2} \succ F(M, 2) / \Sigma_{2} .
$$

Thus there is a commutative diagram of short exact sequences of groups

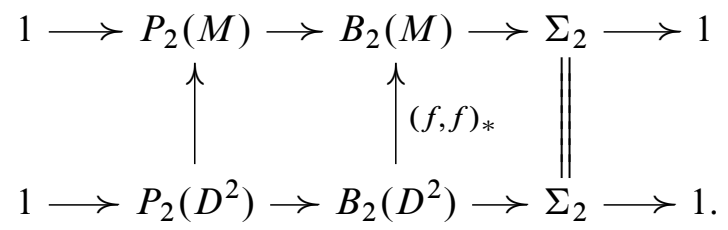

Let $\sigma_{1}$ be a generator for $B_{2}\left(D^{2}\right)=\mathbb{Z}$. Then $(f, f)_{*}\left(\sigma_{1}\right) \neq 1$ in $B_{2}(M)$ as it has nontrivial image in $\Sigma_{2}=B_{2}(M) / P_{2}(M)$. From the commutative diagram

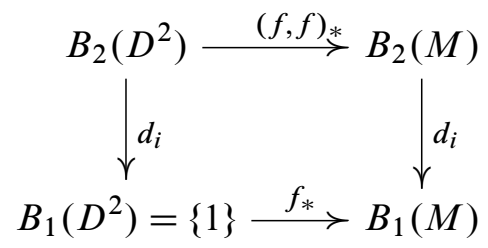

for $i=1,2$, the element $\beta=(f, f)_{*}\left(\sigma_{1}\right)$ is a Brunnian braid on $M$. Let $p_{1}$ be the basepoint of $M$. Choose a loop

$$
\omega:[0,1] \rightarrow M
$$

with $\omega(0)=\omega(1)=p_{1}$ representing a nontrivial element in $\pi_{1}(M)$. Take the second basepoint $p_{2}$ such that $p_{2}$ is not on the curve $\omega([0,1])$ and construct a 2 -strand braid $\gamma$ represented by

$$
\rho(t)=\left\{\rho_{1}(t), \rho_{2}(t)\right\}
$$

with $\rho_{1}(t)=(\omega(t), t)$ and $\rho_{2}(t)=\left(p_{2}, t\right)$ for $0 \leq t \leq 1$ in the cylinder $M \times I$. Then $d_{1}(\gamma)=1$ as represented by the straight line-segment given by $\rho_{2}$, and $d_{2}(\gamma)=[\omega] \neq 1$ is the path homotopy class represented by $\omega$. Observe that $\gamma$ is a pure braid. We have 
$d_{i}\left(\gamma^{-1}\right)=\left(d_{i}(\gamma)\right)^{-1}$. From

$$
\begin{aligned}
d_{1}\left(\gamma \beta \gamma^{-1}\right) & =d_{1}(\gamma) d_{1 \cdot \gamma}(\beta) d_{1 \cdot(\gamma \beta)}\left(\gamma^{-1}\right) \\
& =d_{1}(\gamma) d_{1}(\beta) d_{2}\left(\gamma^{-1}\right) \\
& =d_{1}(\gamma) d_{1}(\beta) d_{2}(\gamma)^{-1} \\
& =1 \cdot 1 \cdot[\omega]^{-1} \\
& \neq 1
\end{aligned}
$$

the conjugate $\gamma \beta \gamma^{-1}$ is not $\operatorname{Brunnian}$ and so $\operatorname{Brun}_{2}(M)$ is not normal. This finishes the proof.

\section{Generating sets for Brunnian braids on surfaces}

In this section, $M$ is a connected compact 2-dimensional (oriented or nonoriented) manifold. The classical Fadell-Neuwirth Theorem will be useful in computations.

Theorem 3.1 [7] The coordinate projection

$$
\delta^{(i)}: F(M, n) \rightarrow F(M, n-1),\left(x_{1}, \ldots, x_{n}\right) \mapsto\left(x_{1}, \ldots, x_{i-1}, x_{i+1}, \ldots, x_{n}\right)
$$

is a fiber bundle with fiber $M \backslash Q_{n-1}$, where $Q_{n-1}$ is a set of $(n-1)$ distinct points in $M$.

Proposition 3.2 Up to a change of basepoint for the pure braid group $P_{n}(M)$ the homomorphism $d_{i}$ coincides with the homomorphism of fundamental groups induced by $\delta^{(i)}$ :

$$
d_{i}=h_{i} \delta_{*}^{(i)}: P_{n}(M) \rightarrow P_{n-1}(M),
$$

where $h_{i}$ is the automorphism of $\pi_{1}(F(M, n-1))$ induced by the change of basepoints

$$
\left(F(M, n-1),\left(p_{1}, \ldots, p_{i-1}, p_{i+1}, \ldots, p_{n}\right)\right) \rightarrow\left(F(M, n-1),\left(p_{1}, \ldots, p_{n-1}\right)\right) .
$$

Let $D^{2}$ be a small disk in $M \backslash \partial M$. The basepoints $\left\{p_{1}, p_{2}, \ldots\right\}$ for the braids on $M$ are chosen inside $D^{2} \backslash \partial D^{2}$. The embedding $f: D^{2} \succ M$ induces a map

$$
f^{n}: F\left(D^{2}, n\right) / \Sigma_{n} \succ F(M, n) / \Sigma_{n}
$$

and so a group homomorphism

$$
f_{*}^{n}: B_{n}\left(D^{2}\right)=\pi_{1}\left(F\left(D^{2}, n\right) / \Sigma_{n}\right) \longrightarrow B_{n}(M)=\pi_{1}\left(F(M, n) / \Sigma_{n}\right)
$$


with a commutative diagram

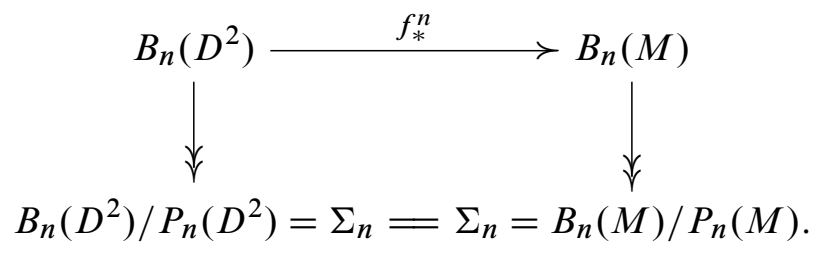

For any braid $\beta \in B_{n}\left(D^{2}\right)$, we write $\beta[M]$ (or simply $\beta$ if there are no confusions) for the braid $f_{*}^{n}(\beta)$ on $M$.

Recall that the Artin braid group $B_{n}\left(D^{2}\right)$ is generated by $\sigma_{1}, \ldots, \sigma_{n-1}$ with defining relations

(1) $\sigma_{i} \sigma_{j}=\sigma_{j} \sigma_{i}$ for $|i-j| \geq 2$;

(2) $\sigma_{i} \sigma_{i+1} \sigma_{i}=\sigma_{i+1} \sigma_{i} \sigma_{i+1}$ for each $i$,

where as a geometric braid, $\sigma_{i}$ is the canonical $i$-th elementary braid of $n$-strands that twists the positions $i$ and $i+1$ once with the $i$ th strand above the $(i+1)$ st and puts the trivial strands on the remaining positions. Also recall that the pure Artin braid group $P_{n}\left(D^{2}\right)$ is generated by

$$
A_{i, j}=\sigma_{j-1} \sigma_{j-2} \cdots \sigma_{i+1} \sigma_{i}^{2} \sigma_{i+1}^{-1} \cdots \sigma_{j-2}^{-1} \sigma_{j-1}^{-1}
$$

for $1 \leq i<j \leq n$.

\subsection{2-Strand Brunnian braids}

Proposition 3.3 Let $M$ be any connected 2-manifold. Then the 2-strand Brunnian braids are determined as follows:

(1) $\operatorname{Brun}_{2}(M) \cap P_{2}(M)$ is the normal closure of the element $A_{1,2}$ in $B_{2}(M)$.

(2) $\operatorname{Brun}_{2}(M)$ is the subgroup of $B_{2}(M)$ generated by $\operatorname{Brun}_{2}(M) \cap P_{2}(M)$ and $\sigma_{1}$, that is $\operatorname{Brun}_{2}(M)=\left\langle\operatorname{Brun}_{2}(M) \cap P_{2}(M), \sigma_{1}\right\rangle$.

Proof (1) Let $\left\langle\left\langle A_{1,2}\right\rangle\right\rangle^{B}$ be the normal closure of $A_{1,2}$ in $B_{2}(M)$. By Proposition 2.4, $\operatorname{Brun}_{2}(M) \cap P_{2}(M)$ is normal in $B_{2}(M)$. Since $A_{1,2}$ is a pure Brunnian braid,

$$
\left\langle\left\langle A_{1,2}\right\rangle\right\rangle^{B} \leq \operatorname{Brun}_{2}(M) \cap P_{2}(M) .
$$


To see the equality, consider the commutative diagram of fiber sequences

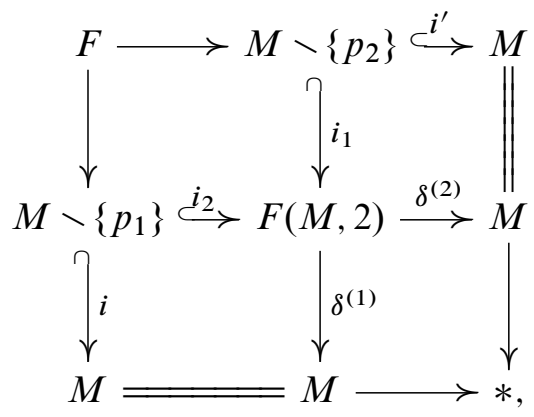

where $i_{2}(x)=\left(p_{1}, x\right)$ and $i_{1}(x)=\left(x, p_{2}\right)$ and $F$ is a homotopy fiber of $i$, which is equivalent to a fiber of $i^{\prime}$. From the middle row, there is an exact sequence

$$
\begin{aligned}
& \pi_{2}(M) \longrightarrow \pi_{1}\left(M \backslash\left\{p_{1}\right\}\right) \stackrel{i_{2 *}}{\longrightarrow} \pi_{1}(F(M, 2)) \\
& =P_{2}(M) \stackrel{d_{2}}{\longrightarrow} \pi_{1}(M)=P_{1}(M)
\end{aligned}
$$

Note that

$\operatorname{Brun}_{2}(M) \cap P_{2}(M)=\operatorname{Ker}\left(d_{1}: P_{2}(M) \rightarrow P_{1}(M)\right) \cap \operatorname{Ker}\left(d_{2}: P_{2}(M) \rightarrow P_{1}(M)\right)$.

Consider the diagram of short exact sequences of groups

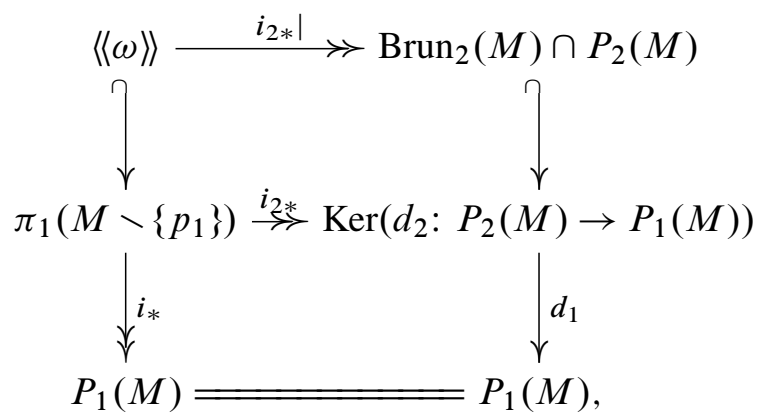

where $\omega \in \pi_{1}\left(M \backslash\left\{p_{1}\right\}\right)$ is represented by a small circle around $p_{1}$. Its commutativity follows from construction and $i_{2 *}$ is an epimorphism by the exact sequence (3-1). It follow from diagram (3-2) that $\operatorname{Brun}_{2}(M) \cap P_{2}(M)$ is the normal closure of $i_{2 *}(\omega)$ in $\operatorname{Ker}\left(d_{2}\right)$. From the commutative diagram

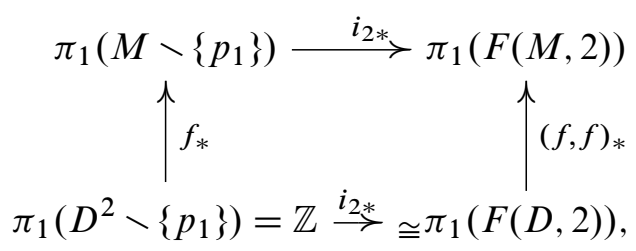


we get

$$
i_{2 *}(\omega)=A_{1,2}^{ \pm 1}
$$

and hence assertion (1) follows.

(2) Note that the braid $\sigma_{1}$ is Brunnian and represents the nontrivial element of $B_{2}(M) / P_{2}(M) \simeq \mathbb{Z} / 2$. From the short exact sequence

$$
1 \rightarrow P_{2}(M) \rightarrow B_{2}(M) \rightarrow \Sigma_{2} \rightarrow 1,
$$

we get the following commutative diagram

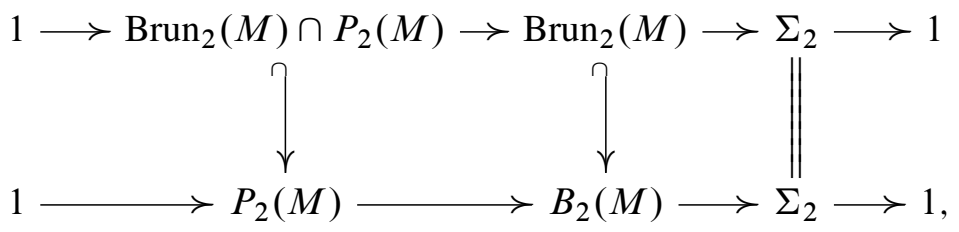

and the assertion follows.

Corollary 3.4 Let $M$ be a connected 2-manifold. Then

$$
B_{2}(M) /\left(\operatorname{Brun}_{2}(M) \cap P_{2}(M)\right)
$$

is the quotient group of $B_{2}(M)$ obtained by adding the single relation

$$
A_{1,2}=\sigma_{1}^{2}=1
$$

\subsection{Homotopy properties of configuration spaces of surfaces}

The following (well-known) fact will be useful for the computations in the next subsections.

Lemma 3.5 Let $M$ be a connected 2-manifold.

(1) If $M \neq S^{2}$ or $\mathbb{R P}^{2}$, then $F(M, n)$ is a $K(\pi, 1)$-space for $n \geq 1$. In particular, $\pi_{2}(F(M, n))=0$ for $n \geq 1$.

(2) $\pi_{2}\left(F\left(S^{2}, n\right)\right)=0$ for $n \geq 3$.

(3) $\pi_{2}\left(F\left(\mathbb{R} P^{2}, n\right)\right)=0$ for $n \geq 2$.

Proof Assertion (1) follows from the fact that $M$ and $M \backslash Q_{n-1}$ are $K(\pi, 1)$ spaces together with Fadell-Neuwirth fibration (Theorem 3.1).

Assertion (2) was proved by Fadell and Van Buskirk [8, Corollary, page 244].

Assertion (3) was proved by Van Buskirk in [22, Corollary, page 82]. 


\subsection{3-Strand Brunnian braids}

We will now determine the 3-strand Brunnian braids on $M$. By [2, Proposition 4.2.2],

$$
\operatorname{Brun}_{n}(M) \subseteq P_{n}(M)
$$

for $n \geq 3$. Thus the determination is given by

$$
\operatorname{Brun}_{n}(M)=\operatorname{Brun}_{n}(M) \cap P_{n}(M)=\bigcap_{i=1}^{n} \operatorname{Ker}\left(d_{i}: P_{n}(M) \rightarrow P_{n-1}(M)\right)
$$

for $n \geq 3$.

For a subset $S$ in $P_{n}(M)$, we write $\langle\langle S\rangle\rangle^{P}$ for the normal closure of $S$ in $P_{n}(M)$, while we keep the notation $\langle\langle S\rangle\rangle$ for the normal closure of $S$ in $B_{n}(M)$.

Proposition 3.6 Let $M$ be a connected 2-manifold. Then the 3-strand Brunnian braids on $M$ are determined as follows:

(1) $\operatorname{Brun}_{3}\left(S^{2}\right)=P_{3}\left(S^{2}\right)=\mathbb{Z} / 2$.

(2) If $M \neq S^{2}$ or $\mathbb{R P}^{2}$, then

$$
\operatorname{Brun}_{3}(M)=\left[\left\langle\left\langle A_{1,3}\right\rangle\right\rangle^{P},\left\langle\left\langle A_{2,3}\right\rangle\right\rangle^{P}\right],
$$

the commutator subgroup of the normal closures in $P_{3}(M)$ of $A_{1,3}$ and $A_{2,3}$, respectively.

Proof Assertion (1) follows directly from the fact that $P_{3}\left(S^{2}\right)=\mathbb{Z} / 2$ (which follows, for example, from [9, Theorem 3.1]) and $P_{2}\left(S^{2}\right)=\{1\}$. For assertion (2), observe that $d_{k} A_{i, j}=1$ for $k=i, j$. Thus

$$
\left\langle\left\langle A_{i, 3}\right\rangle\right\rangle^{P} \leq \operatorname{Ker}\left(d_{3}: P_{3}(M) \rightarrow P_{2}(M)\right) \cap \operatorname{Ker}\left(d_{i}: P_{3}(M) \rightarrow P_{2}(M)\right)
$$

for $i=1,2$ and so the inclusion

$$
\left.\left[\left\langle A_{1,3}\right\rangle\right\rangle^{P},\left\langle\left\langle A_{2,3}\right\rangle\right\rangle^{P}\right] \leq \operatorname{Brun}_{3}(M)
$$

is clear.

From the commutative diagram of the fiber sequences

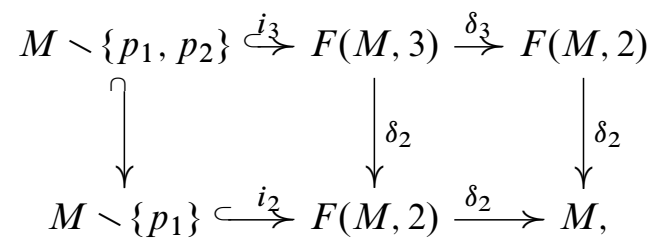


where $i_{3}(x)=\left(p_{1}, p_{2}, x\right)$ and $i_{2}(x)=\left(p_{1}, x\right)$, together with the facts that $\pi_{2}(M)=0$ and $\pi_{2}(F(M, 2))=0$ (Lemma 3.5), there is a commutative diagram of short exact sequences

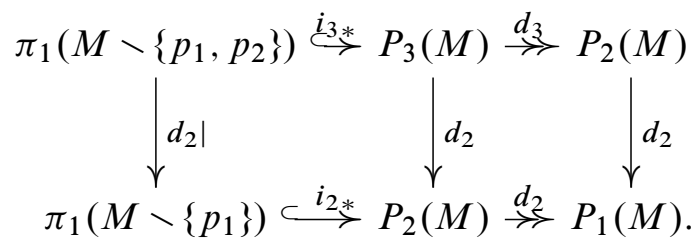

It follows from this diagram that

$$
i_{3 *}: \operatorname{Ker}\left(d_{2} \mid\right) \longrightarrow \operatorname{Ker}\left(d_{3}: P_{3}(M) \rightarrow P_{2}(M)\right) \cap \operatorname{Ker}\left(d_{2}: P_{3}(M) \rightarrow P_{2}(M)\right)
$$

is an isomorphism. Since $d_{2} \mid: \pi_{1}\left(M \backslash\left\{p_{1}, p_{2}\right\}\right) \rightarrow \pi_{1}\left(M \backslash\left\{p_{1}\right\}\right)$ is induced by the inclusion

$$
M \backslash\left\{p_{1}, p_{2}\right\} \subset M \backslash\left\{p_{1}\right\},
$$

$\operatorname{Ker}\left(d_{2} \mid\right)$ is the normal closure of $\left[\omega_{2}\right]$ in $\pi_{1}\left(M \backslash\left\{p_{1}, p_{2}\right\}\right)$, where $\omega_{2}$ is a small circle around $p_{2}$. Similarly, the inclusion

$$
M \backslash\left\{p_{1}, p_{2}\right\} \subset M \backslash\left\{p_{2}\right\}
$$

induces a homomorphism

$$
d_{1} \mid: \pi_{1}\left(M \backslash\left\{p_{1}, p_{2}\right\}\right) \longrightarrow \pi_{1}\left(M \backslash\left\{p_{2}\right\}\right)
$$

with the property that

$$
i_{3 *}: \operatorname{Ker}\left(d_{1} \mid\right) \longrightarrow \operatorname{Ker}\left(d_{3}: P_{3}(M) \rightarrow P_{2}(M)\right) \cap \operatorname{Ker}\left(d_{1}: P_{3}(M) \rightarrow P_{2}(M)\right)
$$

is an isomorphism and $\operatorname{Ker}\left(d_{1} \mid\right)$ is the normal closure of the homotopy class $\left[\omega_{1}\right]$ in $\pi_{1}\left(M \backslash\left\{p_{1}, p_{2}\right\}\right)$, where $\omega_{1}$ is a small circle around $p_{1}$. Thus

$$
i_{3 *}: \operatorname{Ker}\left(d_{1} \mid\right) \cap \operatorname{Ker}\left(d_{2} \mid\right) \longrightarrow \operatorname{Brun}_{3}(M)
$$

is an isomorphism. By applying results of Brown [4] and Brown and Loday [5] to the homotopy pushout diagram of $K(\pi, 1)$-spaces

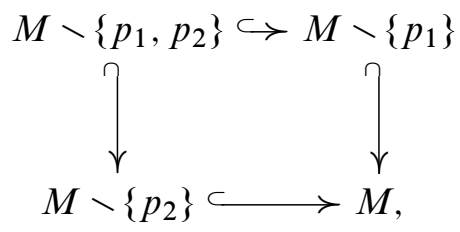


we get an isomorphism

$$
\frac{\operatorname{Ker}\left(d_{1} \mid\right) \cap \operatorname{Ker}\left(d_{2} \mid\right)}{\left[\operatorname{Ker}\left(d_{1} \mid\right), \operatorname{Ker}\left(d_{2} \mid\right)\right]} \cong \pi_{2}(M)=0,
$$

and so,

$$
\operatorname{Ker}\left(d_{1} \mid\right) \cap \operatorname{Ker}\left(d_{2} \mid\right)=\left[\operatorname{Ker}\left(d_{1} \mid\right), \operatorname{Ker}\left(d_{2} \mid\right)\right] .
$$

Together with the isomorphism (3-5) this gives

$$
\operatorname{Brun}_{3}(M)=\left[\left\langle\left\langle i_{3 *}\left(\left[\omega_{1}\right]\right)\right\rangle\right\rangle^{P},\left\langle\left\langle i_{3 *}\left(\left[\omega_{2}\right]\right)\right\rangle\right\rangle^{P}\right] .
$$

Note that the basepoints $\left\{p_{1}, p_{2}\right\}$ are chosen in the interior of the small disk $D^{2}$. From the commutative diagram

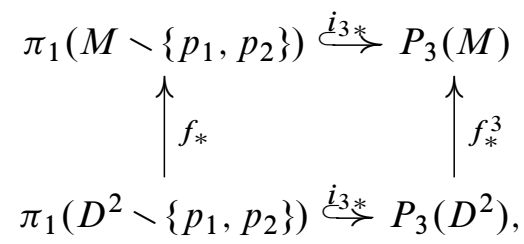

we have $i_{3 *}\left(\left[\omega_{1}\right]\right)=A_{1,3}^{ \pm 1}$ and $i_{3 *}\left(\left[\omega_{2}\right]\right)=A_{2,3}^{ \pm 1}$. Assertion (2) follows from replacing $i_{3 *}\left(\left[\omega_{i}\right]\right)$ by $A_{i, 3}$ in Equation (3-6).

The projective plane case is dealt with separately in Section 4.

\subsection{Colimits of classifying spaces}

Given a group $G$ and its normal subgroups $R_{1}, \ldots, R_{n}$, let us define their complete commutator subgroup as follows

$$
\llbracket R_{1}, R_{2}, \ldots, R_{n} \rrbracket:=\prod_{\substack{I \cup\{1,2, \ldots, n\} \\ I \cap J=\varnothing}}\left[\bigcap_{i \in I} R_{i}, \bigcap_{j \in J} R_{j}\right] .
$$

It is clear that

$$
\llbracket R_{1}, \ldots, R_{n} \rrbracket \subseteq R_{1} \cap \cdots \cap R_{n}
$$

and that the quotient

$$
\frac{R_{1} \cap \cdots \cap R_{n}}{\llbracket R_{1}, R_{2}, \ldots, R_{n} \rrbracket}
$$

is an abelian group with a natural $\mathbb{Z}\left[G / R_{1} \ldots R_{n}\right]$-module structure, where the action is defined via conjugation in $G$. An $n$-tuple of normal subgroups $\left(R_{1}, \ldots, R_{n}\right)$ is 
called connected in $G$ if either $n \leq 2$, or $n \geq 3$ and for all subsets $I, J \subset\{1, \ldots, n\}$ with $|I| \geq 2,|J| \geq 1$ (without the conditions of formula (3-8)) we have the equality

$$
\left(\bigcap_{i \in I} R_{i}\right)\left(\prod_{j \in J} R_{j}\right)=\bigcap_{i \in I}\left(R_{i}\left(\prod_{j \in J} R_{j}\right)\right) .
$$

We will make use of the following result from Ellis and Mikhailov [6]:

Theorem 3.7 Let $G$ be a group, $n \geq 2$, and $\left(R_{1}, \ldots, R_{n}\right)$ an $n$-tuple of normal subgroups in $G$ such that the $(n-1)$-tuples $\left(R_{1}, \ldots, \hat{R}_{i}, \ldots, R_{n}\right)$ are connected for all $1 \leq i \leq n$. Let $X$ be the topological space arising as the colimit of classifying spaces $K\left(G / \prod_{i \in I} R_{i}, 1\right)$, where $I$ ranges over all subsets $I \subsetneq\{1, \ldots, n\}$. Then there is an isomorphism of abelian groups

$$
\pi_{n}(X) \simeq \frac{R_{1} \cap \cdots \cap R_{n}}{\llbracket R_{1}, \ldots, R_{n} \rrbracket} .
$$

\section{5 $n$-Strand Brunnian braids for $n \geq 4$}

Now we are going to determine $\operatorname{Brun}_{n}(M)$ for $n \geq 4$. The case $\operatorname{Brun}_{4}\left(S^{2}\right)$ was determined in [2, Proposition 7.2.2]. Our computation will exclude this special case.

Lemma 3.8 Let $M$ be a connected 2-manifold. Let

$$
d_{k}: P_{n}(M) \rightarrow P_{n-1}(M)
$$

be the operation that removes the $k$-th strand.

(1) Suppose that $M \neq S^{2}$ or $\mathbb{R P}^{2}$. Then, for $n \geq 2$,

$$
\operatorname{Ker}\left(d_{n}\right) \cap \operatorname{Ker}\left(d_{k}\right)=\left\langle\left\langle A_{k, n}\right\rangle\right\rangle^{P}
$$

for $1 \leq k \leq n-1$ and therefore

$$
\operatorname{Brun}_{n}(M)=\bigcap_{k=1}^{n-1}\left\langle\left\langle A_{k, n}\right\rangle\right\rangle^{P} .
$$

Moreover $i_{n *}: \pi_{1}\left(M \backslash\left\{p_{1}, p_{2}, \ldots, p_{n-1}\right\}\right) \rightarrow P_{n}(M)$ is a monomorphism with

$$
i_{n *}\left(\operatorname{Ker}\left(d_{k} \mid\right)\right)=\left\langle\left\langle A_{k, n}\right\rangle\right\rangle^{P},
$$

where $i_{n}$ is given as in (3-3) and

$$
d_{k} \mid: \pi_{1}\left(M \backslash\left\{p_{1}, \ldots, p_{n-1}\right\}\right) \longrightarrow \pi_{1}\left(M \backslash\left\{p_{1}, \ldots, p_{k-1}, p_{k+1}, \ldots, p_{n-1}\right\}\right)
$$

is the group homomorphism induced by inclusion.

(2) If $M=S^{2}$, then the above statement holds for $n \geq 5$.

(3) If $M=\mathbb{R} \mathrm{P}^{2}$, then the above statement holds for $n \geq 4$. 
Proof Diagram (3-3) can be extended to the general case, and so we have the starting commutative diagram for $n \geq 2$ and $1 \leq k \leq n-1$

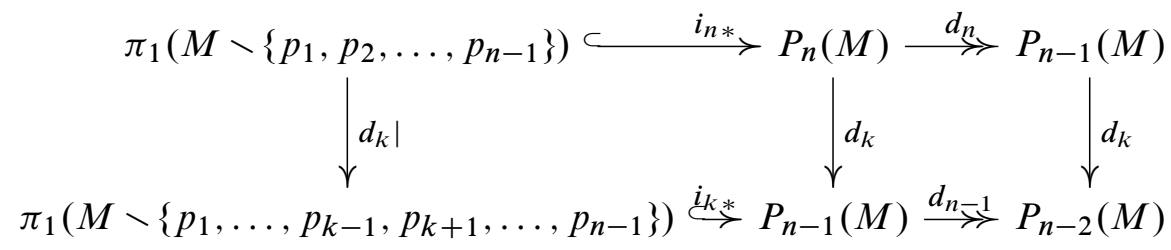

where $i_{k}(x)=\left(p_{1}, \ldots p_{k-1}, x, p_{k+1}, \ldots, p_{n-1}\right)$. Let us consider the homomorphism

$$
i_{k *}: \pi_{1}\left(M \backslash\left\{p_{1}, \ldots, p_{k-1}, p_{k+1}, \ldots, p_{n-1}\right\}\right) \rightarrow P_{n-1}(M) .
$$

It is a monomorphism except 2 cases:

$$
\begin{aligned}
& M=S^{2} \quad \text { and } n=4, \\
& M=\mathbb{R} P^{2} \text { and } n=3 .
\end{aligned}
$$

For $n=2$ this is identical isomorphism. For $n=3$ and $M=S^{2} \pi_{1}\left(M \backslash\left\{p_{i}\right\}\right)$, $i=1,2$, is the trivial group, so $i_{k *}$ is a monomorphism. For the other cases it follows from the exact sequence of the fibration and since

$$
\pi_{2}(F(M, n-2))=0 \text { for } \begin{cases}n \geq 3 & \text { if } M \neq \mathbb{R} P^{2}, S^{2}, \\ n \geq 4 & \text { if } M=\mathbb{R} P^{2}, \\ n \geq 5 & \text { if } M=S^{2}\end{cases}
$$

(by Lemma 3.5). For the exceptional case (3-11) $\pi_{1}\left(S^{2} \backslash\left\{p_{1}, p_{k}, p_{3}\right\}\right)$ is infinite cyclic and $P_{3}\left(S^{2}\right)$ is the cyclic group of order 2. For the exceptional case (3-12) $\pi_{1}\left(\mathbb{R} P^{2} \backslash\left\{p_{i}\right\}\right), i=1,2$, is infinite cyclic and $P_{2}\left(\mathbb{R} P^{2}\right)$ is isomorphic to the finite quaternionic group $\mathbf{Q}_{8}$ [22] (see also Section 4). Thus

$$
i_{n *}: \operatorname{Ker}\left(d_{k} \mid\right) \longrightarrow \operatorname{Ker}\left(d_{n}\right) \cap \operatorname{Ker}\left(d_{k}\right)
$$

is an isomorphism for the cases

$$
\begin{array}{ll}
n \geq 2 & \text { if } M \neq S^{2}, \mathbb{R} \mathrm{P}^{2}, \\
n>3 & \text { if } M=\mathbb{R} P^{2}, \\
n>4 & \text { if } M=S^{2} .
\end{array}
$$

Note that $\operatorname{Ker}\left(d_{k} \mid\right)$ is the normal closure in $\pi_{1}\left(M \backslash\left\{p_{1}, p_{2}, \ldots, p_{n-1}\right\}\right)$ of the homotopy class $\left[\omega_{k}\right]$, where $\omega_{k}$ is a small circle around $p_{k}$. For the same reasons as in 
diagram (3-7), we have $i_{n *}\left(\left[\omega_{k}\right]\right)=A_{k, n}^{ \pm 1}$ and so

$$
\operatorname{Ker}\left(d_{n}\right) \cap \operatorname{Ker}\left(d_{k}\right)=i_{n *}\left(\operatorname{Ker}\left(d_{k} \mid\right)\right) \leq\left\langle\left\langle A_{k, n}\right\rangle\right\rangle^{P} .
$$

On the other hand, $\left\langle\left\langle A_{k, n}\right\rangle\right\rangle^{P} \leq \operatorname{Ker}\left(d_{n}\right) \cap \operatorname{Ker}\left(d_{k}\right)$ because $A_{k, n}$ lies in the normal subgroup $\operatorname{Ker}\left(d_{n}\right) \cap \operatorname{Ker}\left(d_{k}\right)$. Thus, for all cases (3-13)-(3-15),

$$
\operatorname{Ker}\left(d_{n}\right) \cap \operatorname{Ker}\left(d_{k}\right)=\left\langle\left\langle A_{k, n}\right\rangle\right\rangle^{P} .
$$

Hence, the result.

The remaining question is of course how to determine the intersection of the normal subgroups $\left\langle\left\langle A_{k, n}\right\rangle\right\rangle^{P}$ for general $n$. The following result is also given by $\mathrm{Li}$ and $\mathrm{Wu}[17$, Equation (4.1)]. (Note: In [17], the proof was given by checking $K(\pi, 1)$-hypothesis. Our proof is given by checking the connectivity hypothesis in Theorem 3.7.)

Theorem 3.9 Let $M$ be a connected 2-manifold and let $\left\{p_{1}, \ldots, p_{n}\right\}$ be the set of $n$ distinct points in $M \backslash \partial M$. Let

$$
d_{i} \mid: \pi_{1}\left(M \backslash\left\{p_{1}, \ldots, p_{n}\right\}\right) \longrightarrow \pi_{1}\left(M \backslash\left\{p_{1}, \ldots, p_{i-1}, p_{i+1}, \ldots, p_{n}\right\}\right)
$$

be the group homomorphism induced from the inclusion by filling in the missing point $p_{i}$. Then

$$
\left(\bigcap_{i=1}^{n} \operatorname{Ker}\left(d_{i} \mid\right)\right) /\left[\operatorname{Ker}\left(d_{1} \mid\right), \operatorname{Ker}\left(d_{2} \mid\right), \ldots, \operatorname{Ker}\left(d_{n} \mid\right)\right]_{S} \cong \pi_{n}(M)
$$

for each $n \geq 2$.

Proof The surface $M$ can be viewed as a colimit of the spaces $M \backslash \bigsqcup_{i \in I} p_{i}$, where $I$ ranges over all subsets $I \subsetneq\{1, \ldots, n\}$. Denote $G:=\pi_{1}\left(M \backslash\left\{p_{1}, \ldots, p_{n}\right\}\right)$ and $R_{i}:=\operatorname{Ker}\left(d_{i} \mid\right)$. Since punctured surfaces are aspherical, the spaces $M \backslash \bigsqcup_{i \in I} p_{i}$ are classifying spaces for groups $G / \prod_{i \in I} R_{i}$. Let us check that the connectivity condition (3-9) holds for every $(n-1)$-tuple of subgroups $\left(R_{1}, \ldots, \hat{R}_{m}, \ldots, R_{n}\right), 1 \leq m \leq n$. For $n=2,3$, the connectivity condition holds by definition. We prove the statement by induction on $n$. We fix the number $m: 1 \leq m \leq n$, and prove the connectivity (3-9) of the $(n-1)$-tuple $\left(R_{1}, \ldots, \widehat{R}_{m}, \ldots, R_{n}\right)$. Let $I, J \subseteq\{1, \ldots, \hat{m}, \ldots, n\}$. Suppose that $I \cap J \neq \varnothing$. Then the left and right-hand sides of (3-9) are equal to $\prod_{j \in J} R_{j}$ and the condition is proved. So, we can assume that $I \cap J=\varnothing$. Consider the epimorphism

$$
f_{J}: G \rightarrow G / \prod_{j \in J} R_{j}
$$


The condition (3-9) is equivalent to the condition

$$
f_{J}\left(\bigcap_{i \in I} R_{i}\right)=\bigcap_{i \in I} f_{J}\left(R_{i}\right)
$$

Any punctured surface has a free fundamental group and

$$
f_{J}\left(R_{i}\right)=\operatorname{Ker}\left\{\pi_{1}\left(M \backslash \bigsqcup_{k \in I} p_{k}\right) \rightarrow \pi_{1}\left(M \backslash \bigsqcup_{k \in I, k \neq i} p_{k}\right)\right\}
$$

By induction we have

$$
\bigcap_{i \in I} R_{i}=\llbracket R_{i_{1}}, \ldots, R_{i_{|I|}} \rrbracket
$$

for $I=\left\{i_{1}, \ldots, i_{|I|}\right\}$ due to Theorem 3.7 and the fact that punctured surface is aspherical. The same argument shows that

$$
\bigcap_{i \in I} f_{J}\left(R_{i}\right)=\llbracket f_{J}\left(R_{i_{1}}\right), \ldots, f_{J}\left(R_{i_{|I|}}\right) \rrbracket
$$

(we repeat argument for the punctured surface with discs glued to $|J|$ boundary components, the surface remains punctured since $M \backslash\left\{p_{1}, \ldots, p_{n}\right\}$ has at least $n$ boundary components). The same argument shows that

$$
\begin{aligned}
\llbracket R_{i_{1}}, \ldots, R_{i_{|I|}} \rrbracket & =\left[R_{i_{1}}, \ldots, R_{i_{|I|} \mid}\right]_{S}, \\
\llbracket f_{J}\left(R_{i_{1}}\right), \ldots, f_{J}\left(R_{i_{|I|}}\right) \rrbracket & =\left[f_{J}\left(R_{i_{1}}\right), \ldots, f_{J}\left(R_{i_{|I|}}\right)\right]_{S} .
\end{aligned}
$$

Since $f_{J}$ is a homomorphism, the condition (3-16) and hence (3-9) follow. Again observe that

$$
\llbracket R_{1}, R_{2}, \ldots, R_{n} \rrbracket=\left[R_{1}, \ldots, R_{n}\right]_{S},
$$

hence the needed statement follows from Theorem 3.7.

Proof of Theorem 1.1 By Lemma 3.8,

$$
\operatorname{Brun}_{n}(M)=\bigcap_{i=1}^{n-1}\left\langle\left\langle A_{i, n}\right\rangle\right\rangle y^{P}
$$

and $\left\langle\left\langle A_{k, n}\right\rangle\right\rangle y^{P}=i_{n *}\left(\operatorname{Ker}\left(d_{k} \mid\right)\right)$. The assertion follows by Theorem 3.9. 


\section{3-Strand Brunnian braids on the projective plane}

\subsection{Braid group of the projective plane}

There exist several presentations of the group $B_{n}\left(\mathbb{R}^{2}\right)$. See, for example, van Buskirk [22] or Gonçalves and Guaschi [10]. We will use a presentation similar to presentations of the surface braid group from [22].

Theorem 4.1 The group $B_{n}\left(\mathbb{R P}^{2}\right)$ can be presented as having the set of generators

$$
\sigma_{1}, \sigma_{2}, \ldots, \sigma_{n-1}, \rho,
$$

where in the braid $\rho$ the first string represents a nontrivial element of the fundamental group and the rest of the braid is trivial; the generators $\sigma_{1}, \sigma_{2}, \ldots, \sigma_{n-1}$ are the images of classical braid generators of the disk; the set of defining relations is the following:

$$
\begin{array}{rlrl}
\sigma_{i} \sigma_{i+1} \sigma_{i} & =\sigma_{i+1} \sigma_{i} \sigma_{i+1}, & & i=1,2, \ldots, n-2, \\
\sigma_{i} \sigma_{j} & =\sigma_{j} \sigma_{i}, & & |i-j|>1, \\
\rho \sigma_{i} & =\sigma_{i} \rho, & & i \neq 1, \\
\sigma_{1}^{-1} \rho \sigma_{1}^{-1} \rho & =\rho \sigma_{1}^{-1} \rho \sigma_{1}, & \\
\rho^{2} & =\sigma_{1} \sigma_{2} \cdots \sigma_{n-2} \sigma_{n-1}^{2} \sigma_{n-2} \cdots \sigma_{2} \sigma_{1} .
\end{array}
$$

The proof of Theorem 4.1 is given in the Appendix.

Remark 4.2 Geometrically, the element $\rho$ can be depicted similarly to that of [1, Figure 10].

There is a canonical homomorphism $\tau: B_{n}\left(\mathbb{R P}^{2}\right) \longrightarrow \Sigma_{n}, \tau\left(\sigma_{i}\right)=(i, i+1), \tau(\rho)=e$. The kernel, $\operatorname{Ker}(\tau)$, is the pure braid group $P_{n}\left(\mathbb{R P}^{2}\right)$. This group was studied in [10]. We will find a presentation for $P_{3}\left(\mathbb{R} \mathrm{P}^{2}\right)$ which we shall use later. Consider at first the group $B_{2}\left(\mathbb{R} \mathrm{P}^{2}\right)$. We have

$$
B_{2}\left(\mathbb{R P}^{2}\right)=\left\langle\rho, \sigma_{1} \mid \sigma_{1}^{-1} \rho \sigma_{1}^{-1} \rho=\rho \sigma_{1}^{-1} \rho \sigma_{1}, \rho^{2}=\sigma_{1}^{2}\right\rangle .
$$

This group has order 16 and $P_{2}\left(\mathbb{R} \mathrm{P}^{2}\right)$ is isomorphic to the quaternion group $\mathbf{Q}_{8}$ of order 8 [22]. The relation $\rho^{2}=\sigma_{1}^{2}$ gives that $P_{2}\left(\mathbb{R P}^{2}\right)$ is normally generated by $\rho$. Let us define the following element of $P_{2}\left(\mathbb{R P}^{2}\right)$ :

$$
u=\sigma_{1} \rho \sigma_{1}^{-1} .
$$

The Reidemeister-Schreier method (see [18, Theorem 2.9]) gives the presentation

$$
P_{2}\left(\mathbb{R P} \mathrm{P}^{2}\right)=\left\langle\rho, u \mid \rho u \rho=u, \rho^{2}=u^{2}\right\rangle .
$$


This presentation is equivalent to

$$
P_{2}\left(\mathbb{R P} P^{2}\right)=\left\langle\rho, u \mid \rho u \rho=u^{-1}, \rho^{2}=u^{2}\right\rangle,
$$

which appears in Lemma 4.6.

Consider now the case $n=3$. We have

$$
\begin{aligned}
B_{3}\left(\mathbb{R P}^{2}\right)=\left\langle\rho, \sigma_{1}, \sigma_{2}\right| \sigma_{1} \sigma_{2} \sigma_{1}=\sigma_{2} \sigma_{1} \sigma_{2}, & \rho \sigma_{2}=\sigma_{2} \rho, \\
& \left.\sigma_{1}^{-1} \rho \sigma_{1}^{-1} \rho=\rho \sigma_{1}^{-1} \rho \sigma_{1}, \rho^{2}=\sigma_{1} \sigma_{2}^{2} \sigma_{1}\right\rangle .
\end{aligned}
$$

To construct a presentation for $P_{3}\left(\mathbb{R P}^{2}\right)$ we use the Reidemeister-Schreier method. As representatives of cosets of the normal subgroup $P_{3}\left(\mathbb{R} \mathrm{P}^{2}\right)$ in the group $B_{3}\left(\mathbb{R} \mathrm{P}^{2}\right)$ we take the elements: $e, \sigma_{1}, \sigma_{2}, \sigma_{2} \sigma_{1} \sigma_{1} \sigma_{2}, \sigma_{1} \sigma_{2} \sigma_{1}$. Then by [18, Theorem 2.7] the group $P_{3}\left(\mathbb{R} \mathrm{P}^{2}\right)$ is generated by elements

$$
k a \overline{(k a)}^{-1},
$$

where $a \in\left\{\rho, \sigma_{1}, \sigma_{2}\right\}, k \in\left\{e, \sigma_{1}, \sigma_{2}, \sigma_{2} \sigma_{1}, \sigma_{1} \sigma_{2}, \sigma_{1} \sigma_{2} \sigma_{1}\right\}$ and the bar denotes the mapping from words to their coset representatives [18, page 88]. Having in mind that $\sigma_{2} \rho \sigma_{2}^{-1}=\rho$, we obtain that the group $P_{3}\left(\mathbb{R} \mathrm{P}^{2}\right)$ is generated by

$$
\rho, \quad u=\sigma_{1} \rho \sigma_{1}^{-1}, \quad w=\sigma_{2} \sigma_{1} \rho \sigma_{1}^{-1} \sigma_{2}^{-1}, \quad A_{12}, \quad A_{23}=\sigma_{2}^{2}, \quad A_{13}=\sigma_{2} \sigma_{1}^{2} \sigma_{2}^{-1} .
$$

The following set of defining relations is obtained by application of ReidemeisterSchreier method [18, Theorem 2.9]:

$$
\begin{gathered}
A_{12} A_{13} A_{12}^{-1}=A_{23}^{-1} A_{13} A_{23}, \quad A_{12}=\sigma_{1}^{2}\left(A_{13} A_{23}\right) A_{12}^{-1}=A_{13} A_{23}, \\
\rho A_{23} \rho^{-1}=A_{23}, \quad u\left(A_{23}^{-1} A_{13} A_{23}\right) u^{-1}=A_{23}^{-1} A_{13} A_{23}, \\
\rho\left(A_{13}^{-1} w^{-1} A_{13}\right) \rho^{-1}=w^{-1} A_{13}, \quad \rho\left(A_{13}^{-1} w\right) \rho^{-1}=w, \\
\rho\left(A_{12}^{-1} u\right) \rho^{-1}=u, \\
u\left(A_{23}^{-1} w^{-1} A_{23}\right) u^{-1}=w^{-1} A_{23}, \quad u\left(A_{23}^{-1} w\right) u^{-1}=w, \\
A_{23}^{-1} A_{13} A_{23} A_{12}=\rho^{2}, \quad A_{12} A_{13}=\rho^{2}, \quad A_{12} A_{23}=u^{2}, \\
A_{13} A_{23}=w^{2} .
\end{gathered}
$$

From these relations we have the following formulas for conjugation by $A_{12}, \rho, u$ :

$$
A_{12} A_{13} A_{12}^{-1}=A_{23}^{-1} A_{13} A_{23}, \quad A_{12} A_{23} A_{12}^{-1}=A_{23}^{-1} A_{13}^{-1} A_{23} A_{13} A_{23},
$$




$$
\begin{gathered}
A_{12} w A_{12}^{-1}=w, \\
\rho w \rho^{-1}=w^{-1} A_{13}^{-1} w^{2}, \quad \rho A_{13} \rho^{-1}=w^{-1} A_{13}^{-1} w, \\
\rho A_{23} \rho^{-1}=A_{23}, \\
u w u^{-1}=w^{-1} A_{23}^{-1} w^{2}, \quad u A_{23} u^{-1}=w^{-1} A_{23}^{-1} w, \\
u A_{13} u^{-1}=w^{-1} A_{23}^{-1} w A_{23}^{-1} w A_{23} w .
\end{gathered}
$$

Remark 4.3 Relation (4-7) can be more easily seen directly from the relations in $B_{3}\left(\mathbb{R} \mathrm{P}^{2}\right)$. Relations (4-8) are obtained from relations (4-3). Relations (4-9) are obtained from relations (4-4). Relation (4-10) is obtained from relations (4-6) and (4-9).

We see from these formulas that the subgroup

$$
U_{3}\left(\mathbb{R P} \mathrm{P}^{2}\right)=\left\langle w, A_{13}, A_{23} \mid A_{13} A_{23}=w^{2}\right\rangle
$$

is normal in $P_{3}\left(\mathbb{R P}^{2}\right)$. Geometrically, it can be identified with $\pi_{1}\left(\mathbb{R} \mathrm{P}^{2} \backslash\left\{p_{1}, p_{2}\right\}\right)$ which is included in the short exact sequence (see diagram (3-4))

$$
\pi_{1}\left(\mathbb{R} \mathrm{P}^{2} \backslash\left\{p_{1}, p_{2}\right\}\right) \stackrel{i_{3 *}}{\longrightarrow} P_{3}\left(\mathbb{R P}^{2}\right) \stackrel{d_{3}}{\longrightarrow} P_{2}\left(\mathbb{R P}^{2}\right)
$$

and so $U_{3}\left(\mathbb{R} \mathrm{P}^{2}\right)$ is the free group of rank 2 and $P_{3}\left(\mathbb{R} \mathrm{P}^{2}\right) / U_{3}\left(\mathbb{R} \mathrm{P}^{2}\right) \simeq P_{2}\left(\mathbb{R} \mathrm{P}^{2}\right)$.

We can exclude the generators $A_{12}, A_{13}$ from the list of generators for $P_{3}\left(\mathbb{R P}^{2}\right)$, using the formulas

$$
A_{12}=u \rho^{-1} u^{-1} \rho, \quad A_{13}=w^{2} A_{23}^{-1} .
$$

The proof of the following statement is given in the Appendix.

Lemma 4.4 The group $P_{3}\left(\mathbb{R} \mathrm{P}^{2}\right)$ is generated by elements

$$
\rho, \quad u, \quad w, \quad A_{23}
$$

and has the following relations:

$$
\begin{aligned}
\rho w \rho^{-1} & =w^{-1} A_{23}, & & \rho A_{23} \rho^{-1}=A_{23}, \\
\rho^{-1} w \rho & =A_{23} w^{-1}, & \rho^{-1} A_{23} \rho & =A_{23}, \\
u w u^{-1} & =w^{-1} A_{23}^{-1} w^{2}, & u A_{23} u^{-1} & =w^{-1} A_{23}^{-1} w, \\
u^{-1} w u & =A_{23}^{-1} w, & u^{-1} A_{23} u & =A_{23}^{-1} w A_{23}^{-1} w^{-1} A_{23}, \\
\rho^{-1} u \rho^{-1} u^{-1} & =w A_{23}^{-1} w, & u^{-1} \rho^{-1} u^{-1} \rho & =A_{23}^{-1} .
\end{aligned}
$$


Remark 4.5 A similar presentation was constructed in [10, page 765], but in the list of relations there, in the fourth relation of formula (3) instead of

$$
\rho_{2}^{-1} B_{2,3} \rho_{2}=B_{2,3}^{-1} \rho_{3} B_{2,3} \rho_{3}^{-1} B_{2,3},
$$

it should be

$$
\rho_{2}^{-1} B_{2,3} \rho_{2}=B_{2,3}^{-1} \rho_{3} B_{2,3}^{-1} \rho_{3}^{-1} B_{2,3} \text {. }
$$

Let us introduce new generators $a=\rho w, b=w u$. Then we have from Lemma 4.4 the following statement.

Lemma 4.6 The group $P_{3}\left(\mathbb{R} \mathrm{P}^{2}\right)$ can be generated by elements

$$
a, \quad b, \quad w, \quad A_{23}
$$

and has the following relations:

$$
\begin{aligned}
a w a^{-1} & =w^{-1} A_{23}, & a A_{23} a^{-1} & =w^{-1} A_{23} w, \\
a^{-1} w a & =w^{-1} A_{23}, & a^{-1} A_{23} a & =w^{-1} A_{23} w, \\
b w b^{-1} & =A_{23}^{-1} w, & b A_{23} b^{-1} & =A_{23}^{-1}, \\
b^{-1} w b & =A_{23}^{-1} w, & b^{-1} A_{23} b & =A_{23}^{-1}, \\
b a b^{-1} & =a^{-1}, & a^{2} & =b^{2} .
\end{aligned}
$$

In particular, $\langle a, b\rangle \simeq P_{2}\left(\mathbb{R} \mathrm{P}^{2}\right) \leq P_{3}\left(\mathbb{R} \mathrm{P}^{2}\right)$.

From this lemma we have the following statement.

Proposition 4.7 There exists the split short exact sequence

$$
1 \longrightarrow U_{3}\left(\mathbb{R} \mathrm{P}^{2}\right) \longrightarrow P_{3}\left(\mathbb{R} \mathrm{P}^{2}\right) \stackrel{d_{3}}{\longrightarrow} P_{2}\left(\mathbb{R} \mathrm{P}^{2}\right) \longrightarrow 1,
$$

and hence $P_{3}\left(\mathbb{R} \mathrm{P}^{2}\right)=U_{3}\left(\mathbb{R} \mathrm{P}^{2}\right) \lambda P_{2}\left(\mathbb{R} \mathrm{P}^{2}\right)$.

This proposition was proved by Gonçalves and Guaschi [10]. It was also proved there that for $n=2,3$ and for all $m \geq 4$ the short exact sequence

$$
1 \longrightarrow P_{m-n}\left(\mathbb{R} \mathrm{P}^{2} \backslash\left\{x_{1}, x_{2}, \ldots, x_{n}\right\}\right) \longrightarrow P_{m}\left(\mathbb{R} \mathrm{P}^{2}\right) \longrightarrow P_{n}\left(\mathbb{R} \mathrm{P}^{2}\right) \longrightarrow 1
$$

does not split. 


\subsection{3-Strand Brunnian braids on the projective plane}

In order to pass to Brunnian braids recall the geometric interpretations for the generators $\rho, u, w$. We represent $\mathbb{R P}^{2}$ as a 2 -gon $L$ where opposite points on the two edges are identified in the standard manner. In the braid $\rho$, the second and the third strings are just two parallel lines. Its first strand passes through the edge of $L$. The braids $u$ and $w$ are defined in a similar manner. In $u$, the second strand passes through the edge and, in $w$, the third one. The braid $A_{23}$ is defined as in the braid group of a disk. Remember that the presentation for $P_{2}\left(\mathbb{R P}^{2}\right)$ is given by formula (4-1). Hence the maps

$$
d_{1}, d_{2}, d_{3}: P_{3}\left(\mathbb{R} \mathrm{P}^{2}\right) \longrightarrow P_{2}\left(\mathbb{R} \mathrm{P}^{2}\right)
$$

act on the generators by the rules

$$
d_{1}:\left\{\begin{array}{l}
a \longrightarrow u, \\
b \longrightarrow u \rho, \\
A_{23} \longrightarrow A_{12}, \\
w \rightarrow u,
\end{array} \quad d_{2}:\left\{\begin{array}{l}
a \longrightarrow \rho u, \\
b \longrightarrow u, \\
A_{23} \longrightarrow 1, \\
w \longrightarrow u,
\end{array} \quad d_{3}:\left\{\begin{array}{l}
a \longrightarrow \rho, \\
b \longrightarrow u, \\
A_{23} \longrightarrow 1, \\
w \longrightarrow 1
\end{array}\right.\right.\right.
$$

From the exact sequence of Proposition 4.7 we see that $\operatorname{Brun}_{3}\left(\mathbb{R} \mathrm{P}^{2}\right)$ is a subgroup of $U_{3}\left(\mathbb{R} \mathrm{P}^{2}\right)$ and so in our study of Brunnian braids on $\mathbb{R} \mathrm{P}^{2}$ we can restrict ourselves looking at $U_{3}\left(\mathbb{R P}^{2}\right)$ and the action of $d_{1}$ and $d_{2}$ on it. We write the action of $d_{3}$ as supplementary information.

We have

$$
d_{1}\left(w^{4}\right)=d_{2}\left(w^{4}\right)=u^{4}, \quad d_{3}\left(w^{4}\right)=1,
$$

and since $u^{4}=1$ in $P_{2}\left(\mathbb{R} \mathrm{P}^{2}\right)$ then $w^{4} \in \operatorname{Brun}_{3}\left(\mathbb{R} \mathrm{P}^{2}\right)$. Similarly

$$
d_{1}\left(A_{23}^{2}\right)=A_{12}^{2}, \quad d_{2}\left(A_{23}^{2}\right)=d_{3}\left(A_{23}^{2}\right)=1,
$$

and since $A_{12}^{2}=\sigma_{1}^{4}=1$ in $P_{2}\left(\mathbb{R P}^{2}\right)$ (see formula (4-1)), then $A_{23}^{2} \in \operatorname{Brun}_{3}\left(\mathbb{R P}^{2}\right)$. For the commutator $\left[w, A_{23}\right]$ we have

$$
d_{1}\left(\left[w, A_{23}\right]\right)=\left[u, A_{12}\right], \quad d_{2}\left(\left[w, A_{23}\right]\right)=d_{3}\left(\left[w, A_{23}\right]\right)=1,
$$

and $A_{12}$ lies in the center of $P_{2}\left(\mathbb{R} \mathrm{P}^{2}\right)$, so $d_{1}\left(\left[w, A_{23}\right]\right)=1$ and $\left[w, A_{23}\right] \in \operatorname{Brun}_{3}\left(\mathbb{R} \mathrm{P}^{2}\right)$. Now we are going to determine a free basis for $\operatorname{Brun}_{3}\left(\mathbb{R P}^{2}\right)$.

Lemma 4.8 Let $F(S)$ be the free group (freely) generated by the set $S$. Given $x \in S$, let $C_{q}(\bar{x}) \cong \mathbb{Z} / q$ be the cyclic group of order $q$ generated by a formal generator $\bar{x}$. Let $p_{x}: F(S) \rightarrow C_{q}(\bar{x})$ be the group homomorphism with $p(y)=1$ for $y \neq x \in S$ and $p_{x}(x)=\bar{x}$. Then $\operatorname{Ker}\left(p_{x}\right)$ has a free basis

$$
\left\{x^{q}, y,\left[y, x^{j}\right] \mid y \in S, y \neq x, 1 \leq j \leq q-1\right\} .
$$


Proof By using Schreier method, $\operatorname{Ker}\left(p_{x}\right)$ has a free basis

$$
\left\{x^{q}, x^{-j} y x^{j} \mid y \in S, y \neq x, 0 \leq j \leq q-1\right\}
$$

which is equivalent to the generating set in the statement as

$$
\left[y, x^{j}\right]=y^{-1}\left(x^{-j} y x^{j}\right)
$$

and hence the assertion.

Proposition 4.9 As a subgroup of $B_{3}\left(\mathbb{R P}^{2}\right), \operatorname{Brun}_{3}\left(\mathbb{R} \mathrm{P}^{2}\right)$ has a free basis given by

$$
\begin{aligned}
& x_{2}^{2}, \quad x_{1}^{4}, \quad\left[x_{1}^{4}, x_{2}\right], \\
& {\left[x_{2}, x_{1}\right], \quad\left[\left[x_{2}, x_{1}\right], x_{2}\right],} \\
& {\left[x_{2}, x_{1}^{2}\right], \quad\left[\left[x_{2}, x_{1}^{2}\right], x_{2}\right],} \\
& {\left[x_{2}, x_{1}^{3}\right], \quad\left[\left[x_{2}, x_{1}^{3}\right], x_{2}\right] \text {, }}
\end{aligned}
$$

where $x_{1}=w$ and $x_{2}=A_{2,3}$.

Proof Consider the projection $p_{x_{1}}: F\left(x_{1}, x_{2}\right) \rightarrow C_{4}\left(x_{1}\right)$. (It is $d_{2}$ in our case.) By the above lemma, $\operatorname{Ker}\left(p_{x_{1}}\right)$ has a free basis given by

$$
S=\left\{x_{1}^{4}, x_{2},\left[x_{2}, x_{1}\right],\left[x_{2}, x_{1}^{2}\right],\left[x_{2}, x_{1}^{3}\right]\right\} .
$$

The assertion follows by applying the above lemma to the projection $p_{x_{2}}: F\left(x_{1}, x_{2}\right) \rightarrow$ $C_{2}\left(x_{2}\right)\left(d_{1}\right.$ in our case) restricted to the subgroup $F(S)=\operatorname{Ker}\left(p_{x_{1}}\right)$.

Let us describe the quotient groups $P_{3}\left(\mathbb{R} \mathrm{P}^{2}\right) / \operatorname{Brun}_{3}\left(\mathbb{R} \mathrm{P}^{2}\right)$ and $B_{3}\left(\mathbb{R P}^{2}\right) / \operatorname{Brun}_{3}\left(\mathbb{R} \mathrm{P}^{2}\right)$.

Proposition 4.10 (1) Let $\bar{w}$ and $\bar{A}$ be the images of $w$ and $A_{23}$ respectively after applying the natural projection

$$
U_{3}\left(\mathbb{R} \mathrm{P}^{2}\right) \longrightarrow U_{3}\left(\mathbb{R} \mathrm{P}^{2}\right) / \operatorname{Brun}_{3}\left(\mathbb{R} \mathrm{P}^{2}\right) .
$$

Then

$U_{3}\left(\mathbb{R} \mathrm{P}^{2}\right) / \operatorname{Brun}_{3}\left(\mathbb{R} \mathrm{P}^{2}\right)=\left\langle\bar{w}, \bar{A} \mid \bar{w}^{4}=\bar{A}^{2}=1, \bar{A} \bar{w}=\bar{w} \bar{A}\right\rangle \simeq \mathbb{Z}_{4} \oplus \mathbb{Z}_{2}$.

(2) The quotient $P_{3}\left(\mathbb{R} \mathrm{P}^{2}\right) /$ Brun $_{3}\left(\mathbb{R P}^{2}\right)$ has order 64 and is the semidirect product

$$
P_{3}\left(\mathbb{R} \mathrm{P}^{2}\right) / \operatorname{Brun}_{3}\left(\mathbb{R} \mathrm{P}^{2}\right)=\left(U_{3}\left(\mathbb{R} \mathrm{P}^{2}\right) / \operatorname{Brun}_{3}\left(\mathbb{R} \mathrm{P}^{2}\right)\right) \lambda P_{2}\left(\mathbb{R} \mathrm{P}^{2}\right) .
$$

More precisely $P_{3}\left(\mathbb{R} \mathrm{P}^{2}\right) / \mathrm{Brun}_{3}\left(\mathbb{R} \mathrm{P}^{2}\right)$ is generated by

$$
\bar{w}, \quad \bar{A}, \quad a, \quad b
$$


and has defining relations:

$$
\bar{w}^{4}=\bar{A}^{2}=1, \quad \bar{A} \bar{w}=\bar{w} \bar{A}, \quad b a b^{-1}=a^{-1}, \quad a^{2}=b^{2},
$$

$$
\begin{array}{ll}
a^{-1} \bar{w} a=\bar{w}^{-1} \bar{A}, & a^{-1} \bar{A} a=\bar{A}, \\
a \bar{w} a^{-1}=\bar{w}^{-1} \bar{A}, & a \bar{A} a^{-1}=\bar{A}, \\
b^{-1} \bar{w} b=\bar{w} \bar{A}, & b^{-1} \bar{A} b=\bar{A}, \\
b \bar{w} b^{-1}=\bar{w} \bar{A}, & b \bar{A} b^{-1}=\bar{A} .
\end{array}
$$

Proof The first statement follows from Proposition 4.9 and the second statement follows from Proposition 4.9 and Lemma 4.6.

Remark 4.11 The relations without primes are equivalent to those with primes.

Using the short exact sequence

$$
1 \longrightarrow P_{3}\left(\mathbb{R} \mathrm{P}^{2}\right) \longrightarrow B_{3}\left(\mathbb{R} \mathrm{P}^{2}\right) \longrightarrow \Sigma_{3} \longrightarrow 1,
$$

we want to describe $B_{3}\left(\mathbb{R} \mathrm{P}^{2}\right)$ as an extension of $P_{3}\left(\mathbb{R} \mathrm{P}^{2}\right)$ by $\Sigma_{3}$.

Proposition 4.12 The group $B_{3}\left(\mathbb{R P}^{2}\right)$ can be presented as having generators

$$
a, \quad b, \quad w, \quad A_{23}, \quad \sigma_{1}, \sigma_{2},
$$

satisfying relations (4)-(6) from Lemma 4.6 and the following relations:

$$
\begin{aligned}
\sigma_{1} \sigma_{2} \sigma_{1} & =\sigma_{2} \sigma_{1} \sigma_{2} \\
\sigma_{1}^{2} & =a^{2} w^{-2}, \quad \sigma_{2}^{2}=A_{23} . \\
\sigma_{1}^{-1} a \sigma_{1} & =b A_{23}^{-1}, \\
\sigma_{1}^{-1} b \sigma_{1} & =a w^{-1} A_{23} w^{-1}, \\
\sigma_{1}^{-1} w \sigma_{1} & =w \\
\sigma_{1}^{-1} A_{23} \sigma_{1} & =w^{2} A_{23}^{-1}, \\
\sigma_{2}^{-1} a \sigma_{2} & =a b\left(w^{-1} A_{23}\right)^{2}, \\
\sigma_{2}^{-1} b \sigma_{2} & =b A_{23}, \\
\sigma_{2}^{-1} w \sigma_{2} & =b w^{-1} A_{23}, \\
\sigma_{2}^{-1} A_{23} \sigma_{2} & =A_{23} .
\end{aligned}
$$

The proof is given in the Appendix. 
Proposition 4.13 The quotient $B_{3}\left(\mathbb{R P}^{2}\right) / \operatorname{Brun}_{3}\left(\mathbb{R P}^{2}\right)$ has order 384 and is an extension of $P_{3}\left(\mathbb{R} \mathrm{P}^{2}\right) / \mathrm{Brun}_{3}\left(\mathbb{R} \mathrm{P}^{2}\right)$ by $\Sigma_{3}$ :

$$
1 \longrightarrow P_{3}\left(\mathbb{R} P^{2}\right) / \operatorname{Brun}_{3}\left(\mathbb{R} P^{2}\right) \longrightarrow B_{3}\left(\mathbb{R} P^{2}\right) / \operatorname{Brun}_{3}\left(\mathbb{R} P^{2}\right) \longrightarrow \Sigma_{3} \longrightarrow 1 .
$$

The quotient $B_{3}\left(\mathbb{R} \mathrm{P}^{2}\right) / \operatorname{Brun}_{3}\left(\mathbb{R} \mathrm{P}^{2}\right)$ is generated by

$$
\bar{w}, \quad \bar{A}, \quad a, \quad b, \quad \sigma_{1}, \quad \sigma_{2},
$$

and has defining relations:

$$
\begin{gathered}
\bar{w}^{4}=\bar{A}^{2}=1, \quad \bar{A} \bar{w}=\bar{w} \bar{A}, \quad b a b^{-1}=a^{-1}, \quad a^{2}=b^{2}, \\
a^{-1} \bar{w} a=\bar{w}^{-1} \bar{A}, \quad a^{-1} \bar{A} a=\bar{A}, \\
b^{-1} \bar{w} b=\bar{w} \bar{A}, \quad b^{-1} \bar{A} b=\bar{A}, \\
\sigma_{1} \sigma_{2} \sigma_{1}=\sigma_{2} \sigma_{1} \sigma_{2} \\
\sigma_{1}^{2}=a^{2} \bar{w}^{2}, \quad \sigma_{2}^{2}=\bar{A}, \\
\sigma_{1}^{-1} a \sigma_{1}=b \bar{A}, \quad \sigma_{1}^{-1} b \sigma_{1}=a \bar{A}^{2}, \quad \sigma_{1}^{-1} \bar{w} \sigma_{1}=\bar{w}, \quad \sigma_{1}^{-1} \bar{A} \sigma_{1}=\bar{A} \bar{w}^{2}, \\
\sigma_{2}^{-1} a \sigma_{2}=a b \bar{w}^{2}, \quad \sigma_{2}^{-1} b \sigma_{2}=b \bar{A}, \quad \sigma_{2}^{-1} \bar{w} \sigma_{2}=b \bar{A} \bar{w}^{-1}, \quad \sigma_{2}^{-1} \bar{A} \sigma_{2}=\bar{A} .
\end{gathered}
$$

Proof This follows directly from Proposition 4.10(2) and Proposition 4.12.

\section{Proof of Theorem 1.2}

\subsection{Some lemmas on free groups}

Let $S$ be a set and let $F(S)$ be the free group freely generated by $S$. Let $S_{0}$ be a set and let $x_{1}, x_{2}, \ldots$ be additional letters. Let $S_{n}=S_{0} \cup\left\{x_{1}, \ldots, x_{n}\right\}$ be the disjoint union. Consider the group homomorphism

$$
d_{i}: F\left(S_{n}\right) \rightarrow F\left(S_{n-1}\right), \quad 1 \leq i \leq n,
$$

defined by

$$
d_{i}(x)= \begin{cases}x & \text { if } x \in S_{0} \text { or } x=x_{j} \text { with } j<i, \\ 1 & \text { if } x=x_{i}, \\ x_{j-1} & \text { if } x=x_{j} \text { with } j>i .\end{cases}
$$

Roughly speaking, $d_{i}$ is obtained by sending $x_{i}$ to 1 and keeping other generators. The following lemma is a special case of [17, Theorem 4.3]. 
Lemma 5.1 Let $d_{i}: F\left(S_{n}\right) \rightarrow F\left(S_{n-1}\right)$ be defined by the formula (5-1). Then

$$
\bigcap_{j=1}^{k} \operatorname{Ker}\left(d_{i}\right)=\left[\operatorname{Ker}\left(d_{1}\right), \operatorname{Ker}\left(d_{2}\right), \ldots, \operatorname{Ker}\left(d_{k}\right)\right]_{S}
$$

for $2 \leq k \leq n$.

Let $H$ be a normal subgroup of $G$. A set $X$ of elements of $H$ is called a set of normal generators for $H$ in $G$ if $H$ is the normal closure of $X$ in $G$. We say that $H$ has finitely many normal generators in $G$ if there is a finite set $X$ such that $H$ is the normal closure of $X$ in $G$.

Lemma 5.2 Let $R_{1}$ and $R_{2}$ be normal subgroups of $G$. Suppose that

(1) $R_{1}$ has finitely many normal generators;

(2) $R_{2}$ has finitely many generators (in the usual sense).

Then the commutator subgroup $\left[R_{1}, R_{2}\right]$ has finitely many normal generators.

Proof Let $\left\{a_{1}, \ldots, a_{m}\right\}$ be a set of normal generators for $R_{1}$. The set of generators for $R_{1}$ can be given as $\left\{g^{-1} a_{i} g \mid 1 \leq i \leq m, g \in G\right\}$. Let $\left\{b_{1}, \ldots, b_{n}\right\}$ be a set of generators for $R_{2}$. Let $H$ be the normal closure of

$$
\left\{\left[a_{i}, b_{j}\right] \mid 1 \leq i \leq m, 1 \leq j \leq n\right\} .
$$

Now take any $r \in R_{2}, r=b_{i_{1}} \ldots b_{i_{k}}$. Then

$$
\left[a_{i}, r\right]=\left[a_{i}, b_{i_{1}}\right] g_{1}\left[a_{i}, b_{i_{2}}\right] g_{1}^{-1} \cdots g_{j}\left[a_{i}, b_{i_{j+1}}\right] g_{j}^{-1} \cdots g_{k-1}\left[a_{i}, b_{i_{k}}\right] g_{k-1}^{-1},
$$

where $g_{j}=b_{i_{1}} \cdots b_{i_{j}}$. So $\left[a_{i}, r\right] \in H$ for any $r \in R_{2}$. Now

$$
\left[g^{-1} a_{i} g, b_{j}\right]=g^{-1}\left[a_{i}, g b_{j} g^{-1}\right] g \in H,
$$

because $g b_{j} g^{-1} \in R_{2}$. This implies that $\left[R_{1}, R_{2}\right]=H$.

Lemma 5.3 Let $M$ be a connected compact 2-manifold with nonempty boundary. Let $n \geq 2$. Then the subgroup

$$
\bigcap_{i=1}^{k} \operatorname{Ker}\left(d_{i}: P_{n}(M) \rightarrow P_{n-1}(M)\right) \cap \operatorname{Ker}\left(d_{n}: P_{n}(M) \rightarrow P_{n-1}(M)\right)
$$

has finitely many normal generators in $P_{n}(M)$ for each $1 \leq k \leq n-1$. 
Proof The proof is given by induction on $k$. The assertion holds for $k=1$ by Lemma 3.8. Suppose that the assertion holds for $k-1$. Consider the short exact sequence of groups

$$
\pi_{1}\left(M \backslash\left\{p_{1}, \ldots, p_{n-1}\right\}\right) \stackrel{i_{*}}{\longrightarrow} P_{n}(M) \stackrel{d_{n}}{\longrightarrow} P_{n-1}(M) .
$$

Let $\left[\omega_{i}\right] \in \pi_{1}\left(M \backslash\left\{p_{1}, \ldots, p_{n-1}\right\}\right)$ represented by a small circle around $p_{i}$. By Lemma 3.8, for each $1 \leq i \leq n-1$, the subgroup $\operatorname{Ker}\left(d_{i}\right) \cap \operatorname{Ker}\left(d_{n}\right)$ is the normal closure of $\left[\omega_{i}\right]$ in $\pi_{1}\left(M \backslash\left\{p_{1}, \ldots, p_{n-1}\right\}\right)$. Let $R_{i}=\operatorname{Ker}\left(d_{i}\right) \cap \operatorname{Ker}\left(d_{n}\right)$. Note that $\pi_{1}\left(M \backslash\left\{p_{1}, \ldots, p_{n-1}\right\}\right)$ is a free group with a basis containing the elements $\left[\omega_{i}\right]$ for $1 \leq i \leq n-1$. By Lemma 5.1,

$$
\begin{aligned}
\bigcap_{i=1}^{k} R_{i} & =\left[R_{1}, R_{2}, \ldots, R_{k}\right]_{S} \\
& =\prod_{j=1}^{k}\left[\bigcap_{i \in\{1, \ldots, \hat{\jmath}, \ldots, k\}} R_{i}, R_{j}\right]
\end{aligned}
$$

because $R_{i}$ is the kernel of

$$
d_{i} \mid: \pi_{1}\left(M \backslash\left\{p_{1}, \ldots, p_{n-1}\right\}\right) \longrightarrow \pi_{1}\left(M \backslash\left\{p_{1}, \ldots, p_{i-1}, p_{i+1}, \ldots, p_{n-1}\right\}\right)
$$

for $1 \leq i \leq n-1$, and

$$
\bigcap_{i \in\{1, \ldots, \widehat{\jmath}, \ldots, k\}} R_{i}=\left[R_{1}, R_{2}, \ldots, R_{j-1}, R_{j+1}, \ldots, R_{k}\right]_{S} .
$$

It should be noticed also that for normal subgroups $H_{1}, H_{2}, H_{3}$ of a group $G$

$$
\left[H_{1}, H_{3}\right]\left[H_{2}, H_{3}\right]=\left[H_{1} H_{2}, H_{3}\right] \text {, }
$$

see, for example, Serre [21, identity (2’), Proposition 1.1]. It follows that

$$
\begin{aligned}
\bigcap_{i=1}^{k}\left(\operatorname{Ker}\left(d_{i}\right)\right. & \left.\cap \operatorname{Ker}\left(d_{n}\right)\right) \\
& =\prod_{j=1}^{k}\left[\bigcap_{i \in\{1, \ldots, \widehat{J}, \ldots, k\}}\left(\operatorname{Ker}\left(d_{i}\right) \cap \operatorname{Ker}\left(d_{n}\right)\right), \operatorname{Ker}\left(d_{j}\right) \cap \operatorname{Ker}\left(d_{n}\right)\right] \\
& \leq \prod_{j=1}^{k}\left[\bigcap_{i \in\{1, \ldots, \hat{\jmath}, \ldots, k\}}\left(\operatorname{Ker}\left(d_{i}\right) \cap \operatorname{Ker}\left(d_{n}\right)\right), \operatorname{Ker}\left(d_{j}\right)\right] .
\end{aligned}
$$


On the other hand, since

$$
\left[\bigcap_{i \in\{1, \ldots, \widehat{\jmath}, \ldots, k\}}\left(\operatorname{Ker}\left(d_{i}\right) \cap \operatorname{Ker}\left(d_{n}\right)\right), \operatorname{Ker}\left(d_{j}\right)\right] \leq \bigcap_{i=1}^{k}\left(\operatorname{Ker}\left(d_{i}\right) \cap \operatorname{Ker}\left(d_{n}\right)\right),
$$

for every $j=1, \ldots, k$, we have

$$
\bigcap_{i=1}^{k}\left(\operatorname{Ker}\left(d_{i}\right) \cap \operatorname{Ker}\left(d_{n}\right)\right)=\prod_{j=1}^{k}\left[\bigcap_{i \in\{1, \ldots, \hat{j}, \ldots, k\}}\left(\operatorname{Ker}\left(d_{i}\right) \cap \operatorname{Ker}\left(d_{n}\right)\right), \operatorname{Ker}\left(d_{j}\right)\right]
$$

By induction, the subgroup

$$
\bigcap_{i \in\{1, \ldots, \hat{j}, \ldots, k\}}\left(\operatorname{Ker}\left(d_{i}\right) \cap \operatorname{Ker}\left(d_{n}\right)\right)
$$

has finitely many normal generators for every $j=1, \ldots, k$. From the short exact sequence of groups

$$
\pi_{1}\left(M \backslash\left\{p_{1}, \ldots, p_{j-1}, p_{j+1}, \ldots, p_{n}\right\}\right) \hookrightarrow P_{n}(M) \stackrel{d_{k}}{\longleftrightarrow} P_{n-1}(M),
$$

the subgroup $\operatorname{Ker}\left(d_{j}\right)$ has finitely many generators. By Lemma 5.2, the commutator subgroup

$$
\left[\bigcap_{i \in\{1, \ldots, \hat{j}, \ldots, k\}}\left(\operatorname{Ker}\left(d_{i}\right) \cap \operatorname{Ker}\left(d_{n}\right)\right), \operatorname{Ker}\left(d_{j}\right)\right]
$$

has finitely many normal generators for every $j=1, \ldots, k$ and hence the group $\bigcap_{i=1}^{k}\left(\operatorname{Ker}\left(d_{i}\right) \cap \operatorname{Ker}\left(d_{n}\right)\right)$ has finitely many normal generators. The induction is finished.

\subsection{Proof of Theorem 1.2}

The proof is given by two different cases.

Case $1 M$ is a connected compact manifold with nonempty boundary. It is a wellknown fact that groups $P_{n}(M)$ and $B_{n}(M)$ are finitely presented; it can be seen directly using the fibration of Theorem 3.1 and the fact that an extension of finitely presented groups is finitely presented [13, Corollary 2, page 140].

By Lemma 5.3,

$$
\operatorname{Brun}_{n}(M)=\bigcap_{i=1}^{n-1} \operatorname{Ker}\left(d_{i}\right) \cap \operatorname{Ker}\left(d_{n}\right)
$$


has finitely many normal generators in $P_{n}(M)$. This implies the factor groups $P_{n}(M) / \operatorname{Brun}_{n}(M)$ and $B_{n}(M) / \operatorname{Brun}_{n}(M)$ are finitely presented.

Case $2 M$ is a compact closed manifold. Let $\tilde{M}=M \backslash\left\{q_{1}\right\}$. Using the exact sequence of the fibration of Theorem 3.1 and induction on $n$ we conclude that the inclusion $f: \widetilde{M} \rightarrow M$ induces an epimorphism

$$
f_{*}^{n}: P_{n}(\widetilde{M}) \rightarrow P_{n}(M) .
$$

Since

$$
\operatorname{Brun}_{n}(\widetilde{M})=\left[\left\langle\left\langle A_{1, n}\right\rangle\right\rangle y^{P_{n}(\widetilde{M})},\left\langle\left\langle A_{2, n}\right\rangle\right\rangle^{P_{n}(\widetilde{M})}, \ldots,\left\langle\left\langle A_{n-1, n}\right\rangle\right\rangle^{P_{n}(\widetilde{M})}\right]_{S},
$$

we have

$$
f_{*}^{n}\left(\operatorname{Brun}_{n}(\widetilde{M})\right)=\left[\left\langle\left\langle A_{1, n}\right\rangle\right\rangle y^{P_{n}(M)},\left\langle\left\langle A_{2, n}\right\rangle\right\rangle^{P_{n}(M)}, \ldots,\left\langle\left\langle A_{n-1, n}\right\rangle\right\rangle y^{P_{n}(M)}\right]_{S} .
$$

From the fact that $\operatorname{Brun}_{n}(\widetilde{M})$ has finitely many normal generators in $P_{n}(\widetilde{M})$, the subgroup

$$
\left.\left[\left\langle A_{1, n}\right\rangle\right\rangle^{P_{n}(M)},\left\langle\left\langle A_{2, n}\right\rangle\right\rangle^{P_{n}(M)}, \ldots,\left\langle\left\langle A_{n-1, n}\right\rangle\right\rangle^{P_{n}(M)}\right]_{S}
$$

has finitely many normal generators in $P_{n}(M)$.

If $M \neq S^{2}$ or $\mathbb{R P}^{2}$ with $n \geq 3$, then, by Theorem 1.1 and Proposition 3.6, the subgroup

$$
\operatorname{Brun}_{n}(M)=\left[\left\langle\left\langle A_{1, n}\right\rangle\right\rangle y^{P_{n}(M)},\left\langle\left\langle A_{2, n}\right\rangle\right\rangle^{P_{n}(M)}, \ldots,\left\langle\left\langle A_{n-1, n}\right\rangle\right\rangle^{P_{n}(M)}\right]_{S}
$$

has finitely many normal generators in $P_{n}(M)$. Therefore, $P_{n}(M) / \operatorname{Brun}_{n}(M)$ and $B_{n}(M) / \operatorname{Brun}_{n}(M)$ are finitely presented for $M \neq S^{2}$ or $\mathbb{R} \mathrm{P}^{2}$ with $n \geq 3$.

If $M=S^{2}$, then $P_{3}\left(S^{2}\right) / \operatorname{Brun}_{3}\left(S^{2}\right)=\{1\}$ and $B_{3}\left(S^{2}\right) / \operatorname{Brun}_{3}\left(S^{2}\right)=\mathbb{Z} / 2$. For $n=4$, the group $\operatorname{Brun}_{4}\left(S^{2}\right)$ has 5 generators according to [2, Proposition 7.2.1]. Thus $P_{4}\left(S^{2}\right) / \operatorname{Brun}_{4}\left(S^{2}\right)$ and $B_{4}\left(S^{2}\right) / \operatorname{Brun}_{4}\left(S^{2}\right)$ are finitely presented. For $n \geq 5$, by Theorem 1.1, $\operatorname{Brun}_{n}\left(S^{2}\right)$ is a finite extension of the subgroup

$$
\left.\left[\left\langle A_{1, n}\right\rangle\right\rangle y^{P_{n}\left(S^{2}\right)},\left\langle\left\langle A_{2, n}\right\rangle\right\rangle^{P_{n}\left(S^{2}\right)}, \ldots,\left\langle\left\langle A_{n-1, n}\right\rangle\right\rangle^{P_{n}\left(S^{2}\right)}\right]_{S}
$$

because $\pi_{n-1}\left(S^{2}\right)$ is finite. Thus $\operatorname{Brun}_{n}\left(S^{2}\right)$ has finitely many normal generators in $P_{n}\left(S^{2}\right)$ and so the assertion holds for the case $M=S^{2}$.

If $M=\mathbb{R} \mathrm{P}^{2}$, then $\operatorname{Brun}_{3}\left(\mathbb{R} \mathrm{P}^{2}\right)$ has 9 generators according to Proposition 4.9. Thus $P_{3}\left(\mathbb{R} P^{2}\right) / \operatorname{Brun}_{3}\left(\mathbb{R} P^{2}\right)$ and $B_{3}\left(\mathbb{R} P^{2}\right) / \operatorname{Brun}_{3}\left(\mathbb{R} P^{2}\right)$ are finitely presented. For $n \geq 4$, by (3) of Theorem 1.1 together with fact that $\pi_{n-1}\left(S^{2}\right)$ is finitely generated, the subgroup $\operatorname{Brun}_{n}\left(\mathbb{R P}^{2}\right)$ has finitely many normal generators, and so the assertion holds for the case $M=\mathbb{R} \mathrm{P}^{2}$. 


\section{An algorithm for determining a free basis for Brunnian braids}

By Lemma 3.8, in order to get a free basis for $\operatorname{Brun}_{n+1}(M)$, it suffices to determine a free basis for

$$
\bigcap_{i=1}^{n} \operatorname{Ker}\left(d_{i} \mid: \pi_{1}\left(M \backslash\left\{p_{1}, \ldots, p_{n}\right\}\right) \rightarrow \pi_{1}\left(M \backslash\left\{p_{1}, . . i ., p_{n}\right\}\right)\right) .
$$

Let $M$ be connected a $2-$ manifold with nonempty boundary and let $\omega_{i}$ be a small circle around $p_{i}$. Then

$$
\pi_{1}\left(M \backslash\left\{p_{1}, \ldots, p_{n}\right\}\right)=F\left(S_{0} \sqcup\left\{\left[\omega_{1}\right], \ldots,\left[\omega_{n}\right]\right\}\right),
$$

where $\pi_{1}(M)=F\left(S_{0}\right)$. Let $S$ be a set and let $T$ be a subset of $S$. By a projection homomorphism

$$
\pi: F(S) \rightarrow F(T)
$$

we mean here a group homomorphism defined by

$$
\pi(x)= \begin{cases}x & \text { if } x \in T, \\ 1 & \text { if } x \in S \backslash T .\end{cases}
$$

In our case, the homomorphisms $d_{i} \mid$ are projection homomorphisms in the following sense:

Let $S=S_{0} \sqcup\left\{\left[\omega_{1}\right], \ldots,\left[\omega_{n}\right]\right\}$ and let

$$
T_{i}=S_{0} \sqcup\left\{\left[\omega_{1}\right], \ldots,\left[\omega_{i-1}\right],\left[\omega_{i+1}\right], \ldots,\left[\omega_{n}\right]\right\}
$$

for $1 \leq i \leq n$. Then

$$
d_{i} \mid: F(S) \rightarrow F\left(T_{i}\right)
$$

is the projection homomorphism for each $1 \leq i \leq n$. The algorithm in [23, Section 3] provides a recursive formula to determine a free basis for the intersection subgroup $\bigcap_{i=1}^{n} \operatorname{Ker}\left(d_{i} \mid\right)$, as follows. For $x$ a reduced word in the alphabet $S$, and $y$ a reduced word in the alphabet $T$, define $\mu(x, y)$ by induction on the word length of $y$ :

(1) $\mu(x, y)=x$ if $y$ is the empty word;

(2) $\mu(x, y)=\left[\mu\left(x, y^{\prime}\right), z^{\epsilon}\right]$ if $y=y^{\prime} z^{\epsilon}$ with $z \in T$ and $\epsilon= \pm 1$.

Let $V$ be a set of reduced words in the alphabet $S$, and let $W$ be a set of reduced words in the alphabet $T$, a subalphabet of $S$. Define a set of words in the alphabet $S$ :

$$
\mathcal{A}(V)_{W}=\{\mu(x, y) \mid x \in V \text { and } y \in W\} .
$$


By [23, Proposition 3.3], $\mathcal{A}(\{S \backslash T\})_{F(T)}$ is a free basis for the kernel of the projection homomorphism $\pi: F(S) \rightarrow F(T)$. Now for the subsets $T_{1}, \ldots, T_{n}$ of $S$, construct a subset $\mathcal{A}\left(T_{1}, \ldots, T_{k}\right)$ of $F(S)$ by induction on $k$ for $1 \leq k \leq n$ :

(1) $\mathcal{A}\left(T_{1}\right)=\mathcal{A}\left(\left\{S \backslash T_{1}\right\}\right)_{F\left(T_{1}\right)}$.

(2) Let

$T_{2}^{(2)}=\left\{w \in \mathcal{A}\left(T_{1}\right) \mid w=\left[\ldots\left[x, y_{1}^{\epsilon_{1}}\right], \ldots\right], y_{t}^{\epsilon_{t}}\right]$ with $x, y_{j} \in T_{2}, \epsilon_{j}= \pm 1$ for all $\left.j\right\}$ and define

$$
\mathcal{A}\left(T_{1}, T_{2}\right)=\mathcal{A}\left(\mathcal{A}\left(T_{1}\right)\right)_{F\left(T_{2}^{(2)}\right)} .
$$

(3) Suppose $\mathcal{A}\left(T_{1}, \ldots, T_{k-1}\right)$ is defined so every element in $\mathcal{A}\left(T_{1}, \ldots, T_{k-1}\right)$ are written in the form of iterated commutators in $F(S)$ with entries given by \pm powers of elements in $S$. Let

$$
T_{k}^{(k)}=\left\{w \in \mathcal{A}\left(T_{1}, \ldots, T_{k-1}\right) \mid w=\left[x_{1}^{\epsilon_{1}}, \ldots, x_{\ell}^{\epsilon \ell}\right] \text { with } x_{j} \in T_{k} \text { for all } j\right\},
$$

where $\left[x_{1}^{\epsilon_{1}}, \ldots, x_{\ell}^{\epsilon_{\ell}}\right]$ are the elements in $\mathcal{A}\left(T_{1}, \ldots, T_{k-1}\right)$ that are written as iterated commutators. Define

$$
\mathcal{A}\left(T_{1}, \ldots, T_{k}\right)=\mathcal{A}\left(\mathcal{A}\left(T_{1}, \ldots, T_{k-1}\right)\right)_{F\left(T_{k}^{(k)}\right)} .
$$

By [23, Theorem 3.4], $\mathcal{A}\left(T_{1}, \ldots, T_{k}\right)$ is a free basis for $\bigcap_{i=1}^{k} \operatorname{Ker}\left(d_{i} \mid\right)$ for $1 \leq k \leq n$. In particular, $\mathcal{A}\left(T_{1}, \ldots, T_{n}\right)$ is a free basis for $\bigcap_{i=1}^{n} \operatorname{Ker}\left(d_{i} \mid\right)$.

Note In the construction of $\mathcal{A}(V)_{W}$, the words are obtained as iterated commutators with a fixed choice of commutator brackets from left to right. The above algorithm is given by iterating the process of $\mathcal{A}(V)_{W}$ and so the words in $\mathcal{A}\left(T_{1}, \ldots, T_{n}\right)$ are given in the form of iterated commutators with commutator bracket operations given as compositions of left-to-right brackets.

\section{Appendix: Proofs of statements of Section 4}

Proof of Theorem 4.1 We start with the presentation of van Buskirk [22, page 83], also studied in [10]. It has the $2 n-1$ generators $\sigma_{1}, \sigma_{2}, \ldots, \sigma_{n-1}, \rho_{1}, \ldots, \rho_{n}$, subject to the following relations:

$$
\begin{aligned}
\sigma_{i} \sigma_{i+1} \sigma_{i} & =\sigma_{i+1} \sigma_{i} \sigma_{i+1}, & & i=1,2, \ldots, n-2, \\
\sigma_{i} \sigma_{j} & =\sigma_{j} \sigma_{i}, & & |i-j|>1, \\
\rho_{j} \sigma_{i} & =\sigma_{i} \rho_{j}, & & j \neq i, i+1,
\end{aligned}
$$




$$
\begin{aligned}
\rho_{i} & =\sigma_{i} \rho_{i+1} \sigma_{i}, \\
\rho_{i+1}^{-1} \rho_{i}^{-1} \rho_{i+1} \rho_{i} & =\sigma_{i}^{2} \\
\rho_{1}^{2} & =\sigma_{1} \sigma_{2} \cdots \sigma_{n-2} \sigma_{n-1}^{2} \sigma_{n-2} \cdots \sigma_{2} \sigma_{1} .
\end{aligned}
$$

Let us show at first that the system (i)-(vi) is equivalent to the system (i)-(iv), (vi) and the relations

$$
\sigma_{i}^{-1} \rho_{i} \sigma_{i}^{-1} \rho_{i}=\rho_{i} \sigma_{i}^{-1} \rho_{i} \sigma_{i}, \quad i=1, \ldots, n-1
$$

We multiply the equality (7-1) by $\sigma_{i} \rho_{i}^{-1} \sigma_{i} \rho_{i}^{-1}$ on the left-hand side and we obtain

$$
\sigma_{i} \rho_{i}^{-1} \sigma_{i} \rho_{i}^{-1} \sigma_{i}^{-1} \rho_{i} \sigma_{i}^{-1} \rho_{i}=\sigma_{i}^{2}, \quad i=1, \ldots, n-1
$$

Then we use the expression

$$
\rho_{i+1}=\sigma_{i}^{-1} \rho_{i} \sigma_{i}^{-1}
$$

from (iv) and we obtain (v). Hence, the relations (7-1) hold in $B_{n}\left(\mathbb{R P}^{2}\right)$.

Now we show by induction that we can eliminate all the equalities in (7-1) except the first one, ie for $i=1$,

$$
\sigma_{1}^{-1} \rho_{1} \sigma_{1}^{-1} \rho_{1}=\rho_{1} \sigma_{1}^{-1} \rho_{1} \sigma_{1}
$$

In other words we will show that relations (7-1) for $i=2, \ldots, n-1$ are consequences of relations (i)-(iv) and (7-2). For $i=2$ we start with (7-2) and multiply it by $\sigma_{1}^{-1} \sigma_{2}^{-1}$ on the left-hand side and by $\sigma_{2}^{-1} \sigma_{1}^{-1}$ on the right-hand side. We get

$$
\sigma_{1}^{-1} \sigma_{2}^{-1} \sigma_{1}^{-1} \rho_{1} \sigma_{1}^{-1} \rho_{1} \sigma_{2}^{-1} \sigma_{1}^{-1}=\sigma_{1}^{-1} \sigma_{2}^{-1} \rho_{1} \sigma_{1}^{-1} \rho_{1} \sigma_{1} \sigma_{2}^{-1} \sigma_{1}^{-1}
$$

We apply relations (i) to this relation on the right-hand side and on the left-hand side, we obtain

$$
\sigma_{2}^{-1} \sigma_{1}^{-1} \sigma_{2}^{-1} \rho_{1} \sigma_{1}^{-1} \rho_{1} \sigma_{2}^{-1} \sigma_{1}^{-1}=\sigma_{1}^{-1} \sigma_{2}^{-1} \rho_{1} \sigma_{1}^{-1} \rho_{1} \sigma_{2}^{-1} \sigma_{1}^{-1} \sigma_{2}
$$

Further we apply relation (iii) to permute $\rho_{1}$ and $\sigma_{2}^{-1}$ in all four appearances of $\rho_{1}$ in the last relation, we get

$$
\sigma_{2}^{-1} \sigma_{1}^{-1} \rho_{1} \sigma_{2}^{-1} \sigma_{1}^{-1} \sigma_{2}^{-1} \rho_{1} \sigma_{1}^{-1}=\sigma_{1}^{-1} \rho_{1} \sigma_{2}^{-1} \sigma_{1}^{-1} \sigma_{2}^{-1} \rho_{1} \sigma_{1}^{-1} \sigma_{2}
$$

Now apply relation (i) to the middle parts of both sides of the last relation, and obtain

$$
\sigma_{2}^{-1} \sigma_{1}^{-1} \rho_{1} \sigma_{1}^{-1} \sigma_{2}^{-1} \sigma_{1}^{-1} \rho_{1} \sigma_{1}^{-1}=\sigma_{1}^{-1} \rho_{1} \sigma_{1}^{-1} \sigma_{2}^{-1} \sigma_{1}^{-1} \rho_{1} \sigma_{1}^{-1} \sigma_{2}
$$

Use relation (iv) in the form $\rho_{2}=\sigma_{1}^{-1} \rho_{1} \sigma_{1}^{-1}$ and obtain

$$
\sigma_{2}^{-1} \rho_{2} \sigma_{2}^{-1} \rho_{2}=\rho_{2} \sigma_{2}^{-1} \rho_{2} \sigma_{2} \text {. }
$$


This is relation (7-1) for $i=2$. Suppose now that for $i$ our statement is true: the relation

$$
\sigma_{i}^{-1} \rho_{i} \sigma_{i}^{-1} \rho_{i}=\rho_{i} \sigma_{i}^{-1} \rho_{i} \sigma_{i}
$$

is a consequence of relations (i)-(iv) and (7-2). Multiplying this relation by $\sigma_{i}^{-1} \sigma_{i+1}^{-1}$ on the left-hand side and by $\sigma_{i+1}^{-1} \sigma_{i}^{-1}$ on the right-hand side and applying relations (i)-(iv) as before we obtain relation (7-1) for $i+1$. So all relations (v) can be replaced by one relation (7-2).

Let us consider, now, relations (iii) and show that all of them are consequences of relations (i), (ii), (iv) and relations

$$
\rho_{1} \sigma_{i}=\sigma_{i} \rho_{1}, \quad i \neq 1 .
$$

Let $j>1$, then it follows from (iv) that

$$
\rho_{j}=\sigma_{j-1}^{-1} \sigma_{j-2}^{-1} \cdots \sigma_{1}^{-1} \rho_{1} \sigma_{1}^{-1} \cdots \sigma_{j-2}^{-1} \sigma_{j-1}^{-1} .
$$

Consider $\sigma_{i} \rho_{j}$. Let $i<j-1$, then using relations (i), (ii) and (7-3) we have

$$
\begin{aligned}
\sigma_{i} \rho_{j} & =\sigma_{i} \sigma_{j-1}^{-1} \sigma_{j-2}^{-1} \cdots \sigma_{1}^{-1} \rho_{1} \sigma_{1}^{-1} \cdots \sigma_{j-2}^{-1} \sigma_{j-1}^{-1} \\
& =\sigma_{j-1}^{-1} \sigma_{j-2}^{-1} \cdots \sigma_{1}^{-1} \sigma_{i+1} \rho_{1} \sigma_{1}^{-1} \cdots \sigma_{j-2}^{-1} \sigma_{j-1}^{-1} \\
& =\sigma_{j-1}^{-1} \sigma_{j-2}^{-1} \cdots \sigma_{1}^{-1} \rho_{1} \sigma_{i+1} \sigma_{1}^{-1} \cdots \sigma_{j-2}^{-1} \sigma_{j-1}^{-1} \\
& =\sigma_{j-1}^{-1} \sigma_{j-2}^{-1} \cdots \sigma_{1}^{-1} \rho_{1} \sigma_{1}^{-1} \cdots \sigma_{j-2}^{-1} \sigma_{j-1}^{-1} \sigma_{i}=\rho_{j} \sigma_{i} .
\end{aligned}
$$

If $i>j$, then using relations (i) and (7-3) we have

$$
\begin{aligned}
\sigma_{i} \rho_{j} & =\sigma_{i} \sigma_{j-1}^{-1} \sigma_{j-2}^{-1} \cdots \sigma_{1}^{-1} \rho_{1} \sigma_{1}^{-1} \cdots \sigma_{j-2}^{-1} \sigma_{j-1}^{-1} \\
& =\sigma_{j-1}^{-1} \sigma_{j-2}^{-1} \cdots \sigma_{1}^{-1} \sigma_{i} \rho_{1} \sigma_{1}^{-1} \cdots \sigma_{j-2}^{-1} \sigma_{j-1}^{-1} \\
& =\sigma_{j-1}^{-1} \sigma_{j-2}^{-1} \cdots \sigma_{1}^{-1} \rho_{1} \sigma_{i} \sigma_{1}^{-1} \cdots \sigma_{j-2}^{-1} \sigma_{j-1}^{-1} \\
& =\sigma_{j-1}^{-1} \sigma_{j-2}^{-1} \cdots \sigma_{1}^{-1} \rho_{1} \sigma_{1}^{-1} \cdots \sigma_{j-2}^{-1} \sigma_{j-1}^{-1} \sigma_{i}=\rho_{j} \sigma_{i} .
\end{aligned}
$$

Hence all relations (iii) are consequences of relations (i), (ii), (iv) and (7-3). So, we can delete generators $\rho_{2}, \ldots, \rho_{n}$, and relations (iv) from the presentation and replace relations (iii) and (v) by relations (7-3) and (7-2) respectively.

Proof of Lemma 4.4 Relations $\left(1^{\prime}\right)$ and $\left(2^{\prime}\right)$ follow from relations (1) and (2) respectively. Relation (1) follows from (4-8), and relation (2) is (4-9). The first relation in (3) follows from (4-11), the second relation in (4-5), and the second relation in (4-8). The second relation in (3) follows from (4-11) and the third relation in (4-5). To prove that the statement of the lemma gives a presentation of $P_{3}\left(\mathbb{R P}^{2}\right)$, denote by $P$ the 
group which has a presentation given by these generators and relations. There exists an evident homomorphism

$$
\phi: P \rightarrow P_{3}\left(\mathbb{R} \mathrm{P}^{2}\right) .
$$

The subgroup $U_{3}\left(\mathbb{R} \mathrm{P}^{2}\right)$ generated by $w$ and $A_{2,3}$ is a free subgroup in $P$ as it is free after the mapping by $\phi$. It can be seen that the quotient $P / U_{3}\left(\mathbb{R P}^{2}\right)$ is isomorphic to $P_{2}\left(\mathbb{R P}^{2}\right)$ (relations (3)); so $\phi$ becomes an isomorphism after comparison of exact sequences:

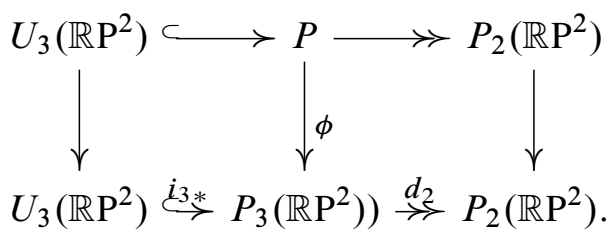

This completes the proof.

Proof of Proposition 4.12 The first relation in (4-13) follows from the definition of the elements $a$ and $w$, and the relations of the presentation of $B_{3}\left(\mathbb{R P}^{2}\right)$ with generators $\rho, \sigma_{1}$ and $\sigma_{2}$. The second relation in (4-13) is the definition of $A_{23}$.

To construct the formulas of conjugation we can take the corresponding relations from the paper of van Buskirk [22] and rewrite them in our generators of $P_{3}\left(\mathbb{R P}^{2}\right)$. We can also prove these formulas using the relations that we already know to hold in $B_{3}\left(\mathbb{R} P^{2}\right)$. Let us do it. At first let us prove (4-17). We start with the two equal expressions for $A_{13}$ :

$$
\sigma_{1}^{-1} \sigma_{2}^{2} \sigma_{1}=\sigma_{2} \sigma_{1}^{2} \sigma_{2}^{-1},
$$

which is true in $B_{3}\left(\mathbb{R P}^{2}\right)$. We insert $\sigma_{2} \sigma_{2}^{-1}$ in the right-hand part of (7-4):

$$
\sigma_{1}^{-1} \sigma_{2}^{2} \sigma_{1}=\sigma_{2} \sigma_{1}^{2} \sigma_{2} \sigma_{2}^{-2} \text {. }
$$

Then we use the relation $\rho^{2}=\sigma_{1} \sigma_{2}^{2} \sigma_{1}$ from the presentation of $B_{3}\left(\mathbb{R P}^{2}\right)$ :

$$
\sigma_{1}^{-1} \sigma_{2}^{2} \sigma_{1}=\sigma_{2} \sigma_{1} \rho^{2} \sigma_{1}^{-1} \sigma_{2}^{-1} A_{23}^{-1} \text {. }
$$

Since $A_{23}=\sigma_{2}^{2}$ and $w=\sigma_{2} \sigma_{1} \rho \sigma_{1}^{-1} \sigma_{2}^{-1}$, we have

$$
\sigma_{1}^{-1} A_{23} \sigma_{1}=w^{2} A_{23}^{-1} \text {. }
$$

To prove relation (4-16), we start with the definition of $w$ :

$$
\sigma_{2} \sigma_{1} \rho \sigma_{1}^{-1} \sigma_{2}^{-1}=w
$$

Since $\rho \sigma_{2}=\sigma_{2} \rho$ we have

$$
\sigma_{2} \sigma_{1} \rho \sigma_{1}^{-1} \sigma_{2}^{-1}=\left(\sigma_{2} \sigma_{1} \sigma_{2}^{-1}\right) \rho\left(\sigma_{2} \sigma_{1}^{-1} \sigma_{2}^{-1}\right)=\left(\sigma_{1}^{-1} \sigma_{2} \sigma_{1}\right) \rho\left(\sigma_{1}^{-1} \sigma_{2}^{-1} \sigma_{1}\right)=\sigma_{1}^{-1} w \sigma_{1} \text {, }
$$


and so

$$
\sigma_{1}^{-1} w \sigma_{1}=w
$$

To prove relation (4-15), we start with relation $\left(1^{\prime}\right)$ from Lemma 4.4

$$
\rho^{-1} w \rho=A_{23} w^{-1}
$$

which is equivalent to

$$
w \rho=\rho A_{23} w^{-1} .
$$

Since $\sigma_{1}^{-1} w \sigma_{1}=w$ and $\sigma_{1}^{-1} u \sigma_{1}=\rho$ we have

$$
\sigma_{1}^{-1} w u \sigma_{1}=(\rho w) w^{-1} A_{23} w^{-1} .
$$

Using the definition of $a$ and $b, a=\rho w, b=w u$, we obtain relation (4-15). For relation (4-14), we start with the equality

$$
w=\left(A_{23} w^{-1}\right)\left(w A_{23}^{-1} w\right)
$$

and apply the conjugation formulas $\left(1^{\prime}\right)$ and (3) from Lemma 4.4. This gives

$$
w=\left(\rho^{-1} w \rho\right)\left(\rho^{-1} u \rho^{-1} u^{-1}\right),
$$

which is equivalent to

$$
w=\rho^{-1} w u \rho^{-1} u^{-1} .
$$

We rewrite the first equation in (3) from Lemma 4.4 in the form

$$
\rho\left(w A_{23}^{-1} w\right) u \rho u^{-1}=1
$$

and multiplying the right-hand side of (7-5) by $\rho\left(w A_{23}^{-1} w\right) u \rho u^{-1}$, we obtain

$$
w=\rho^{-1} w u \rho^{-1} u^{-1} \rho\left(w A_{23}^{-1} w\right) u \rho u^{-1} .
$$

We apply (1) of Lemma 4.4 and we get

$$
w=\rho^{-1} w u \rho^{-1} u^{-1} \rho\left(\rho A_{23} w^{-2} \rho^{-1}\right) u \rho u^{-1} .
$$

Using the formulas

$$
A_{12}=u \rho^{-1} u^{-1} \rho, \quad A_{13}^{-1}=A_{23} w^{-2},
$$

we obtain

$$
w=\rho^{-1} w A_{12} \rho A_{13}^{-1} A_{12}^{-1} \quad \text { or } \quad \rho w=w A_{12} \rho A_{13}^{-1} A_{12}^{-1} .
$$

Conjugating it by $\sigma_{1}^{-1}$ we have

$$
\sigma_{1}^{-1}(\rho w) \sigma_{1}=w u A_{23}^{-1},
$$

which is (4-14). 
Formula (4-21) follows from $A_{23}=\sigma_{2}^{2}$.

To prove relation (4-20), we start with the first relation in $\left(2^{\prime}\right)$ of Lemma 4.4 and we rewrite it in equivalent forms

$$
u^{-1} w u=A_{23}^{-1} w \Leftrightarrow 1=u^{-1} w u w^{-1} A_{23} \Leftrightarrow u=w u w^{-1} A_{23}
$$

or

$$
\sigma_{2}^{-1}\left(\sigma_{2} \sigma_{1} \rho \sigma_{1}^{-1} \sigma_{2}^{-1}\right) \sigma_{2}=w u w^{-1} A_{23}
$$

which is equivalent to (4-20):

$$
\sigma_{2}^{-1} w \sigma_{2}=b w^{-1} A_{23} .
$$

For relation (4-19), we start with the identity

$$
\left(b w^{-1} A_{23}\right)\left(A_{23}^{-1} w A_{23}\right)=b A_{23} .
$$

Using the formula

$$
\sigma_{2}^{-1} u \sigma_{2}=A_{23}^{-1} w A_{23},
$$

and (4-20), we get

$$
\left(\sigma_{2}^{-1} w \sigma_{2}\right)\left(\sigma_{2}^{-1} u \sigma_{2}\right)=b A_{23} .
$$

This is equivalent to (4-19):

$$
\sigma_{2}^{-1}(w u) \sigma_{2}=b A_{23} .
$$

Finally let us prove relation (4-18). We start with the identity

$$
1=\left(A_{23}^{-1} w\right) w^{-1} A_{23} .
$$

Using $\left(2^{\prime}\right)$ of Lemma 4.4 we get

$$
1=\left(u^{-1} w u\right) w^{-1} A_{23}
$$

and then

$$
u=(w u) w^{-1} A_{23} .
$$

We multiply this equality by $\rho w$ from the left-hand side

$$
\rho w u=(\rho w)(w u) w^{-1} A_{23},
$$

which is equivalent to

$$
\rho b=a b w^{-1} A_{23} .
$$

Since $\rho$ and $\sigma_{2}$ commute, this is the same as

$$
\left(\sigma_{2}^{-1} \rho \sigma_{2}\right) b=a b w^{-1} A_{23} \text {. }
$$


Multiply this equality by $w^{-1} A_{23}$ from the right-hand side

$$
\left(\sigma_{2}^{-1} \rho \sigma_{2}\right)\left(b w^{-1} A_{23}\right)=a b\left(w^{-1} A_{23}\right)^{2},
$$

and use (4-20)

$$
\sigma_{2}^{-1} \rho w \sigma_{2}=a b\left(w^{-1} A_{23}\right)^{2}
$$

which is equivalent to (4-18):

$$
\sigma_{2}^{-1} a \sigma_{2}=a b\left(w^{-1} A_{23}\right)^{2} .
$$

The proof that $B_{3}\left(\mathbb{R P}^{2}\right)$ has a presentation as in the statement of the Proposition is the same as the proof of the presentation of Lemma 4.4 with the help of the exact sequence (4-12).

Acknowledgements This work was started during the visit of Jie Wu to the University Montpellier 2 in December 2003, and it was continued during the visit of V V Vershinin to the National University of Singapore in July 2007. The main results were obtained during the stay of the four authors in Oberwolfach in the framework of the program RiP during May 18-June 7, 2008. The authors would like to thank the Mathematical Institute of Oberwolfach for its hospitality. The work was continued by the last two authors during the visit of the third author in the Institute for Mathematical Sciences of the National University of Singapore during the program on Algebraic Topology, Braids and Mapping Class Groups December 4-19, 2008. He would like to express his gratitude to the IMS.

The last author is partially supported by the AcRF Tier 1 (WBS number R-146-000137-112) and AcRF Tier 2 (WBS number R-146-000-143-112) of MOE of Singapore and NSFC grant number 11028104.

The third author is thankful to Ronnie Brown for his comments on the generalized van Kampen Theorem.

Authors are thankful to Kent Orr and the anonymous reviewer for their valuable comments and suggestions to improve the manuscript.

\section{References}

[1] P Bellingeri, On presentations of surface braid groups, J. Algebra 274 (2004) 543-563 MR2043362

[2] A J Berrick, F R Cohen, Y L Wong, J Wu, Configurations, braids, and homotopy groups, J. Amer. Math. Soc. 19 (2006) 265-326 MR2188127 
[3] J S Birman, Braids, links, and mapping class groups, Annals of Math. Studies 82, Princeton Univ. Press (1974) MR0375281

[4] R Brown, Coproducts of crossed P-modules: applications to second homotopy groups and to the homology of groups, Topology 23 (1984) 337-345 MR770569

[5] R Brown, J-L Loday, Van Kampen theorems for diagrams of spaces, Topology 26 (1987) 311-335 MR899052 With an appendix by M Zisman

[6] G Ellis, R Mikhailov, A colimit of classifying spaces, Adv. Math. 223 (2010) 20972113 MR2601009

[7] E Fadell, L Neuwirth, Configuration spaces, Math. Scand. 10 (1962) 111-118 MR0141126

[8] E Fadell, J Van Buskirk, The braid groups of $E^{2}$ and $S^{2}$, Duke Math. J. 29 (1962) 243-257 MR0141128

[9] R Gillette, J Van Buskirk, The word problem and consequences for the braid groups and mapping class groups of the 2-sphere, Trans. Amer. Math. Soc. 131 (1968) 277296 MR0231894

[10] D L Gonçalves, J Guaschi, The braid groups of the projective plane, Algebr. Geom. Topol. 4 (2004) 757-780 MR2100679

[11] G G Gurzo, The group of smooth braids, from: "XVI All-Union Algebra Conference Part 2 (Leningrad, 1981)”, (A P Oskolkov, editor), Akad. Nauk SSSR Mat. Inst. Leningrad. Otdel., Leningrad (1981) 39-40 MR698950 In Russian

[12] D L Johnson, Towards a characterization of smooth braids, Math. Proc. Cambridge Philos. Soc. 92 (1982) 425-427 MR677467

[13] D L Johnson, Presentations of groups, second edition, London Math. Soc. Student Texts 15, Cambridge Univ. Press (1997) MR1472735

[14] H Levinson, Decomposable braids and linkages, Trans. Amer. Math. Soc. 178 (1973) 111-126 MR0324684

[15] H Levinson, Decomposable braids as subgroups of braid groups, Trans. Amer. Math. Soc. 202 (1975) 51-55 MR0362287

[16] J Y Li, J Wu, Artin braid groups and homotopy groups, Proc. Lond. Math. Soc. 99 (2009) 521-556 MR2551462

[17] J Y Li, J Wu, On symmetric commutator subgroups, braids, links and homotopy groups, Trans. Amer. Math. Soc. 363 (2011) 3829-3852 MR2775829

[18] W Magnus, A Karrass, D Solitar, Combinatorial group theory: Presentations of groups in terms of generators and relations, Wiley Interscience, New York-LondonSydney (1966) MR0207802 
[19] G S Makanin, Question 6.23, from: "Kourovka Notebook (Unsolved Questions in Group Theory)", (V D Mazurov, editor), seventh edition, Akad. Nauk SSSR Sibirsk. Otdel. Inst. Mat., Novosibirsk (1980) 78 MR617914

[20] R Mikhailov, I B S Passi, J Wu, Symmetric ideals in group rings and simplicial homotopy, J. Pure Appl. Algebra 215 (2011) 1085-1092 MR2747240

[21] J-P Serre, Lie algebras and Lie groups, second edition, Lecture Notes in Math. 1500, Springer, Berlin (1992) MR1176100 1964 lectures given at Harvard University

[22] J van Buskirk, Braid groups of compact 2-manifolds with elements of finite order, Trans. Amer. Math. Soc. 122 (1966) 81-97 MR0189013

[23] J Wu, Combinatorial descriptions of homotopy groups of certain spaces, Math. Proc. Cambridge Philos. Soc. 130 (2001) 489-513 MR1816806

Sobolev Institute of Mathematics

Novosibirsk, 630090, Russia

Steklov Mathematical Institute

Gubkina 8, Moscow, 119991, Russia

Département des Sciences Mathématiques, Université Montpellier II Place Eugène Bataillon, 3, 34095 Montpellier cedex 5, France (and Sobolev Institute of Mathematics)

Department of Mathematics, National University of Singapore 2 Science Drive 2, Singapore 117542, Singapore

bardakov@math.nsc.ru, rmikhailov@mail.ru, vershini@math.univ-montp2.fr, matwuj@nus.edu.sg http://www. math. nus.edu.sg/ matwujie

Received: 18 December 2011 Revised: 6 May 2012 